\title{
The $I_{K s}$ Ion Channel Activator Mefenamic Acid Requires KCNE1 and Modulates Channel Gating in a Subunit-Dependent Manner\$s
}

\author{
Yundi Wang, Jodene Eldstrom, and David Fedida \\ Department of Anesthesiology, Pharmacology and Therapeutics, University of British Columbia, Vancouver, British Columbia, \\ Canada
}

Received July 19, 2019; accepted November 4, 2019

\section{ABSTRACT}

The pairing of KCNQ1 and KCNE1 subunits together mediates the cardiac slow delayed rectifier current $\left(I_{K s}\right)$, which is partly responsible for cardiomyocyte repolarization and physiologic shortening of the cardiac action potential. Mefenamic acid, a nonsteroidal anti-inflammatory drug, has been identified as an $I_{K s}$ activator. Here, we provide a biophysical and pharmacological characterization of mefenamic acid's effect on $I_{K s}$. Using whole-cell patch clamp, we show that mefenamic acid enhances $I_{K s}$ activity in both a dose- and stoichiometry-dependent fashion by changing the slowly activating and deactivating $I_{K s}$ current into an almost linear current with instantaneous onset and slowed tail current decay, sensitive to the $I_{K s}$ blocker (3R,4S)-(+)-N-[3-hydroxy-2,2-dimethyl-6-(4,4,4-trifluorobutoxy) chroman-4-yl]- $N$-methylmethanesulfonamide (HMR1556). Both single channels, which reveal no change in the maximum conductance, and whole-cell studies, which reveal a dramatically altered conductance-voltage relationship despite increasingly longer interpulse intervals, suggest mefenamic acid decreases the voltage sensitivity of the $I_{K s}$ channel and shifts channel gating kinetics toward more negative potentials. Modeling studies revealed that changes in voltage sensor activation kinetics are sufficient to reproduce the dose and frequency dependence of mefenamic acid action on $I_{K S}$ channels. Mutational analysis showed that mefenamic acid's effect on $I_{K s}$ required residue $\mathrm{K} 41$ and potentially other surrounding residues on the extracellular surface of KCNE1, and explains why the KCNQ1 channel alone is insensitive to up to $1 \mathrm{mM}$ mefenamic acid. Given that mefenamic acid can enhance all $I_{K S}$ channel complexes containing different ratios of $K C N Q 1$ to $K C N E 1$, it may provide a promising therapeutic approach to treating life-threatening cardiac arrhythmia syndromes.

\section{SIGNIFICANCE STATEMENT}

The channels which generate the cardiac slow delayed rectifier $\mathrm{K}^{+}$current $\left(I_{K S}\right)$ are composed of KCNQ1 and KCNE1 subunits. Due to the critical role played by $I_{K s}$ in heartbeat regulation, enhancing $I_{K s}$ current has been identified as a promising therapeutic strategy to treat various heart rhythm diseases. Most $I_{K s}$ activators, unfortunately, only work on KCNQ1 alone and not the physiologically relevant $I_{K_{s}}$ channel. We have demonstrated that mefenamic acid can enhance $I_{K s}$ in a dose- and stoichiometrydependent fashion, regulated by its interactions with KCNE1.

\section{Introduction}

The potassium voltage-gated KCNQ channel subfamily is composed of five known isoforms, KCNQ1-5 (Abbott, 2014). Expression of the first isoform, KCNQ1, has been detected throughout the body, including in the heart, stomach, and ear (Liin et al., 2015). When by itself, KCNQ1 produces a fast activating and deactivating current that has not yet been found to underlie any specific endogenous currents in the body (Abbott, 2014). KCNQ1, however, also coassembles with several $\beta$-subunits, KCNE1-5, which modulate KCNQ1

This research was funded by the Natural Sciences and Engineering Research Council of Canada [Grant RGPIN-2016-05422], the Canadian Institutes of Health Research [Grant PJT-156181], and the Heart and Stroke Foundation of Canada [Grant G17-0018392] (grants to D.F.). Y.W. holds a Canadian Institutes of Health Research-Frederick Banting and Charles Best Canada Graduate scholarship.

https://doi.org/10.1124/mol.119.117952.

S This article has supplemental material available at molpharm.aspetjournals.org. current kinetics (Bendahhou et al., 2005; Manderfield and George, 2008; Eldstrom and Fedida, 2011). In the heart, the coassembly of KCNQ1 with KCNE1, and perhaps other KCNE subunits, produces a slowly activating and deactivating cardiac delayed rectifier $\mathrm{K}^{+}$current $\left(I_{K s}\right)$, which contributes significantly to cardiac repolarization (Sanguinetti et al., 1996; Lundquist et al., 2005).

There is no general agreement on the stoichiometric ratio of KCNE1 to KCNQ1 subunits underlying $I_{K s}$, either in vivo or in heterologous in vitro expression systems (Morin and Kobertz, 2008; Nakajo et al., 2010; Plant et al., 2014; Murray et al., 2016), although we know a variable stoichiometry of $4: 1$ up to 4:4 is possible (Murray et al., 2016). Given that the kinetics of $I_{K s}$ are greatly affected by the number of KCNE1 subunits, great flexibility in the expressed physiologic and pharmacological properties of $I_{K s}$ channel complexes is expected from a variable stoichiometry.

ABBREVIATIONS: DIDS, 4,4'-diisothiocyanato-2,2'-stilbenedisulfonic acid; $\mathrm{EC}_{\max }$, maximal effective concentration; G-V, conductance-voltage relationship; HMR1556, (3R,4S)-(+)- $N$-[3-hydroxy-2,2-dimethyl-6-(4,4,4-trifluorobutoxy) chroman-4-yl]- $N$-methylmethanesulfonamide; $I_{K s}$, cardiac slow delayed rectifier $\mathrm{K}^{+}$current; $I_{\max }-I_{\min }$, peak to steady-state difference currents; $I_{\min }$, minimum current amplitude; I-V, current-voltage relationship; $k$, slope factor; LM, Itk-mouse fibroblast; LQTS, long QT syndrome; $n^{H}$, Hill coefficient; $\mathrm{V}_{1 / 2}$, voltage at half-maximal activation; VS, voltage sensor; WT, wild type. 
The complex also has clinical importance in disease syndromes, including cardiac arrhythmia, with the severity ranging from syncope to sudden death (Splawski et al., 2000). Approximately $50 \%$ of the mutations seen in long QT syndrome (LQTS) patients are in the KCNQ1 subunit (LQTS type 1) (Hedley et al., 2009), with mutations in KCNE1 causing LQTS type 5.

Activators of $I_{K s}$ that can act on the relevant saturated and unsaturated $I_{K s}$ complexes are of particular interest, as they may have therapeutic potential in the treatment of LQTS types 1 and 5. To date, although several activators have been reported, some are only effective on KCNQ1 alone with limited efficacy on $I_{K s}$. These include ML-277, zinc pyrithione, and L364,373 (Magyar et al., 2006; Gao et al., 2008; Yu et al., 2013). The known activators of $I_{K s}$ are phenylboronic acid (Mruk and Kobertz, 2009) and hexachlorophene (Zheng et al., 2012), as well as stilbenes such as 4,4'-diisothiocyanato-2,2' -stilbenedisulfonic acid (DIDS) and 4-acetamido- $4^{\prime}$-isothiocyanatostilbene-2,2' -disulfonic acid, fenamates such as mefenamic acid (Abitbol et al., 1999), and fatty acids such as lauric acid (Doolan et al., 2002). Phenylboronic acid and hexachlorophene have been shown to increase both KCNQ1 alone and $I_{K s}$ current amplitudes, although they are more potent on $I_{K s}$ (Mruk and Kobertz, 2009; Zheng et al., 2012). In contrast, lauric acid, DIDS, and 4-acetamido-4' -isothiocyanatostilbene$2,2^{\prime}$-disulfonic acid have been shown to only increase $I_{K s}$ current (Abitbol et al., 1999; Doolan et al., 2002).

The fenamate mefenamic acid (Fig. 1A) is a nonsteroidal anti-inflammatory drug primarily prescribed to treat menstrual pains (https:/www.accessdata.fda.gov/drugsatfda_docs/ label/2008/015034s040lbl.pdf). Originally identified as a chloride channel blocker, mefenamic acid has since been shown in various expression systems (Xenopus oocytes, rat mesenteric arteries, canine ventricular myocytes, guinea pig ventricular myocytes, CHO cells, and COS-7 cells) to increase mammalian $I_{K s}$ current amplitudes as well as produce a variable amount of instantaneous current and inhibit tail current decay (Busch et al., 1994; Abitbol et al., 1999; Unsöld et al., 2000; Magyar et al., 2006; Toyoda et al., 2006; Chadha et al., 2012).

In the present study, using transiently expressed human $I_{K s}$ in mammalian cells, we have carried out a more complete biophysical characterization of the effects of mefenamic acid than has been attempted to date. We show that mefenamic acid has a minimal effect on KCNQ1 in the absence of KCNE1 and have quantified drug concentration- and rate-dependent changes in the $I_{K s}$ current waveforms, the conductancevoltage relationship, and single-channel conductance and kinetics. Because the stoichiometry of $I_{K s}$ may vary and affect its pharmacology and current kinetics (Nakajo et al., 2010; Murray et al., 2016), we have analyzed the dependence of mefenamic acid actions on the stoichiometry of $I_{K s}$ channel complexes. Last, through mutational analysis, we identify a specific regulatory site for mefenamic acid on KCNE1. The results suggest that residue $\mathrm{K} 41$ on $\mathrm{KCNE} 1$ is of particular importance in mediating the effect mefenamic acid has on $I_{K s}$.

\section{Materials and Methods}

\section{Solutions and Drugs}

Unless otherwise stated, all drugs and chemicals used to make solutions were obtained from Sigma-Aldrich (Mississauga, ON, Canada). The control bath solution for whole-cell experiments contained $135 \mathrm{mM} \mathrm{NaCl}, 5 \mathrm{mM} \mathrm{KCl}, 1 \mathrm{mM} \mathrm{MgCl}, 2.8 \mathrm{mM} \mathrm{NaAcetate}$, and
$10 \mathrm{mM}$ HEPES, pH 7.4 with $\mathrm{NaOH}$. The pipette solution for whole-cell experiments contained $130 \mathrm{mM} \mathrm{KCl}, 5 \mathrm{mM}$ EGTA, $1 \mathrm{mM} \mathrm{MgCl}_{2}, 4 \mathrm{mM}$ $\mathrm{Na}_{2}$-ATP, $0.1 \mathrm{mM}$ GTP, and $10 \mathrm{mM}$ HEPES, $\mathrm{pH} 7.2$ with KOH. The control bath solution for single-channel experiments contained $135 \mathrm{mM} \mathrm{KCl}, 1 \mathrm{mM} \mathrm{MgCl} 2,50 \mu \mathrm{M} \mathrm{CaCl}_{2}, 10 \mathrm{mM}$ dextrose, and $10 \mathrm{mM}$ HEPES, $\mathrm{pH} 7.4$ with $\mathrm{KOH}$. The pipette solution for singlechannel experiments contained $6 \mathrm{mM} \mathrm{NaCl}, 129 \mathrm{mM}$ 2-(4-morpholino)-ethane sulfonic acid, $1 \mathrm{mM} \mathrm{MgCl}_{2}, 5 \mathrm{mM} \mathrm{KCl}$, and $10 \mathrm{mM}$ HEPES, pH 7.4 with $\mathrm{NaOH}$. Mefenamic acid and HMR1556 ((3R,4S)(+)- $N$-[3-hydroxy-2,2-dimethyl-6-(4,4,4-trifluorobutoxy) chroman-4yl]- $N$-methylmethanesulfonamide; Tocris Bioscience, Oakville, ON, Canada) were prepared as stock solutions $(50,200$, or $500 \mathrm{mM}$ for mefenamic acid and $2 \mathrm{mM}$ for HMR1556) dissolved in 100\% dimethylsulfoxide. Stock mefenamic acid solutions were diluted in control whole-cell bath solution to obtain final mefenamic acid concentrations of $10,30,100,300,500 \mu \mathrm{M}$, or $1 \mathrm{mM}$, which were perfused onto mammalian cells for whole-cell experiments. Stock HMR1556 and mefenamic acid solutions were pipetted directly into the chamber to obtain a final HMR1556 concentration of $1 \mu \mathrm{M}$ for whole-cell experiments or a final mefenamic acid concentration of $100 \mu \mathrm{M}$ for singlechannel experiments. Concentrations of dimethylsulfoxide in final bath solutions never exceeded $0.2 \%(\mathrm{v} / \mathrm{v})$. The maximum concentration of mefenamic acid ( $1 \mathrm{mM}$ ) lowered the $\mathrm{pH}$ of the final bath solution by $0.15 \pm 0.02(n=3)$. This was not corrected.

\section{Constructs, Cell Culture, and Transfection}

$I_{K s}$ is generally understood to be functionally composed of combinations of KCNQ1 and KCNE1 subunits. The stoichiometry of the two subunits may be variable in heterologous expression systems (Murray et al., 2016) and may also vary in vivo (Dvir et al., 2014). In the initial experiments (Figs. 1-4), the initial stoichiometric ratio of KCNQ1: KCNE1 was set at the maximum, 4:4. This was achieved through transfection of a linked KCNE1 and KCNQ1 cDNA (Murray et al., 2016), which is expected to assemble as a tetramer with four KCNQ1 and four KCNE1 subunits. For simplicity, this will be denoted as EQ. In later experiments where the ratio was varied (Fig. 5), cells were transfected with KCNQ1 without KCNE1 (Q1), linked constructs containing one KCNE1 linked with two KCNQ1s (EQQ, expected to assemble in a 2:4 ratio), or one KCNE1 linked with four KCNQ1s (EQQQQ, expected to assemble in a 1:4 ratio). EQ, EQQ, and EQQQQ constructs were generated as previously described (Murray et al., 2016). In all cases, we consider the currents that result from different combinations of KCNQ1 and KCNE1 (except KCNQ1 alone, Q1) to be $I_{K s}$, and we use this name interchangeably with the constructs themselves.

tsA201 transformed human embryonic kidney 293 or ltk-mouse fibroblast cells were cultured in modified Eagle's medium supplemented with $10 \%$ fetal calf serum and $100 \mu \mathrm{g} / \mathrm{ml}$ penicillin, $100 \mu \mathrm{g} / \mathrm{ml}$ streptomycin, and $0.25 \mu \mathrm{g} / \mathrm{ml}$ amphotericin and plated for whole-cell and single-channel experiments, as previously described (Murray et al., 2016; Westhoff et al., 2019). Cells were transiently transfected with: 1) GFP-tagged Q1 (Q1-GFP); 2) EQ, EQQ, or EQQQQ and GFP in a 1.5-2.5:1.0- $\mu$ g ratio; or 3) mutant KCNE1 and Q1-GFP in a 4.5-6.0: 1.5- $\mu$ g ratio using Lipofectamine2000 (Thermo Fisher Scientific, Waltham, MA) as per the manufacturer's protocol. All KCNE1 mutations were generated using site-directed mutagenesis and Pfu Turbo (Agilent Technologies, Santa Clara, CA) followed by sequence confirmation of all mutations. Whole-cell and single-channel experiments were conducted 24-48 hours post transfection.

\section{Electrophysiology}

An Axopatch 200B amplifier, Digidata 1440A (whole-cell experiments), or Digidata 1322A (single-channel experiments) and pClamp 9 or 11 software (all from Molecular Devices, San Jose, CA) were used to conduct all experiments. For whole-cell experiments, electrodes ranging from 1- to $3-\mathrm{M} \Omega$ resistance were first pulled from thin-walled borosilicate glass (Sutter Instrument, Novato, CA) using a linear multistage electrode puller (Sutter Instrument) and then fire polished 
before use. Series resistance compensation of 70\%-80\% was used for all whole-cell experiments. Data were sampled at $20 \mathrm{kHz}$ and filtered at $5 \mathrm{kHz}$ during acquisition. Electrodes for single-channel experiments were pulled from thick-walled borosilicate glass (Sutter Instrument) and fire polished to resistances between 40 and $60 \mathrm{M} \Omega$. Before recording, electrodes were coated with Sylgard (Dow Corning, Midland, MI). Current records were sampled at $10 \mathrm{kHz}$; low-pass filtered at $2 \mathrm{kHz}$ at acquisition using a $-3-\mathrm{dB}$, four-pole Bessel filter; and digitally filtered at $200 \mathrm{~Hz}$ for presentation and analysis (Werry et al., 2013; Eldstrom et al., 2015; Murray et al., 2016; Thompson et al., 2017; Westhoff et al., 2017). For voltage clamp protocols, interpulse intervals refer to start-to-start times between sweeps.

\section{Data Analysis}

GraphPad Prism 8.1.1 software (GraphPad Software) was used to analyze all data. Where applicable, one-way ANOVA followed by the
Bonferroni multiple comparison post hoc test was used to determine statistical significance. A $P$ value less than 0.05 was considered statistically significant. All data in the figures are shown as means \pm S.E.M. and reported in the tables as means \pm S.D.

Whole-Cell Experiments. Mefenamic acid dose-response diary plots (Fig. 1, B and C, right panels) and curves (Figs. 2, A and B; Fig. 5D) were obtained from measurement of the activating $I_{K s}$ current. Specifically, the peak to steady-state difference currents $\left(\mathrm{I}_{\max }\right.$ $-I_{\text {min }}$ ) were calculated by subtracting the minimum amplitude of the activating current $\left(\mathrm{I}_{\text {min }}\right)$ from the peak amplitude of the activating current $\left(I_{\max }\right)$. This value was then plotted against either the corresponding sweep number or $\log _{10}$ concentration of mefenamic acid (Fig. 1; Fig. 2A). Where applicable, the difference current in mefenamic acid was normalized to the maximum control (in the absence of mefenamic acid) difference current and subtracted from 1.0 to obtain the "normalized response," which was plotted against the
A

Mefenamic Acid (Mef)

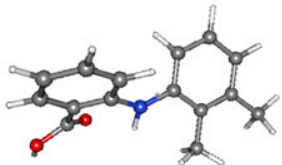

B

\section{Untransfected}

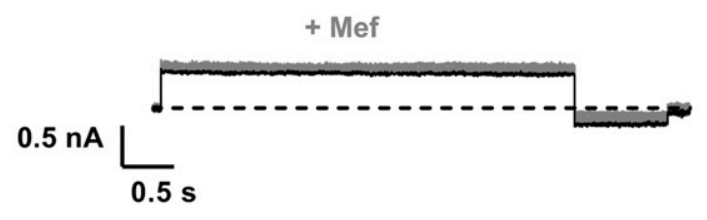

C $\quad E Q$

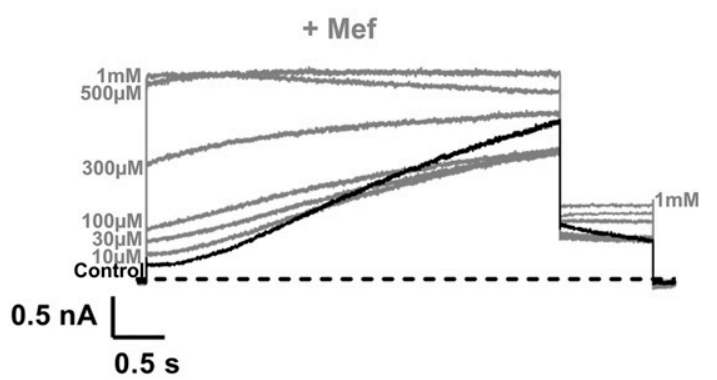

D

$E Q$

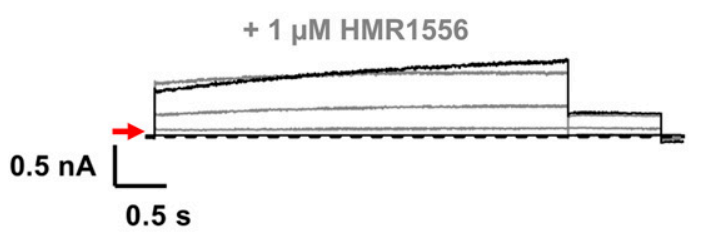

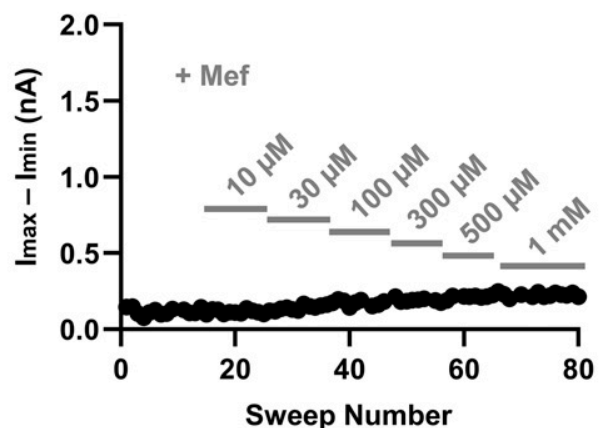
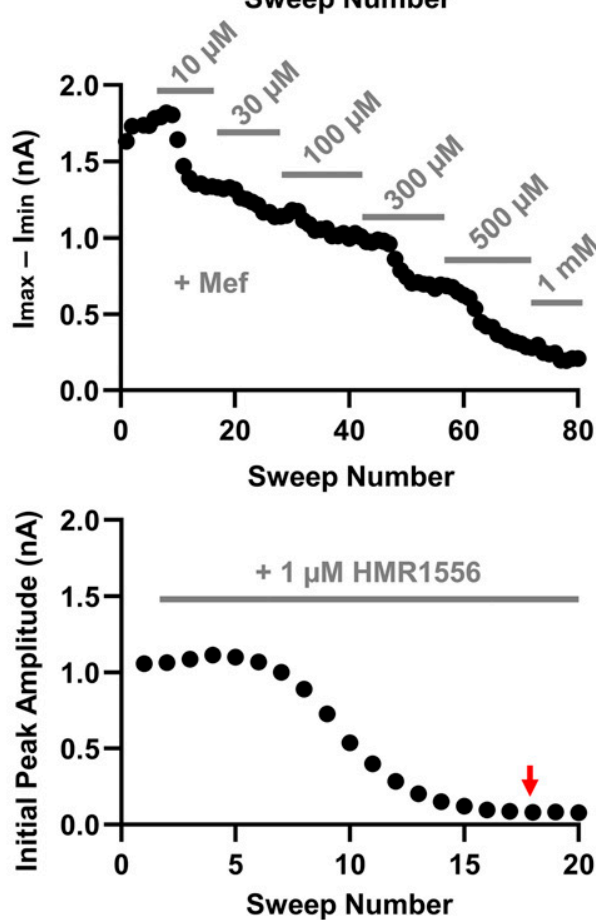

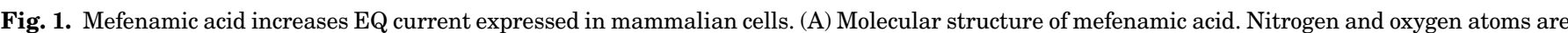

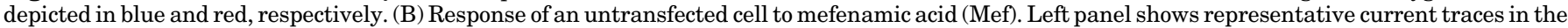

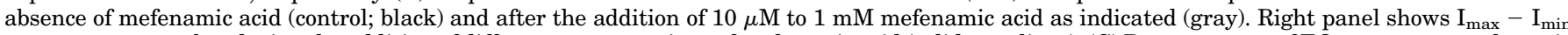

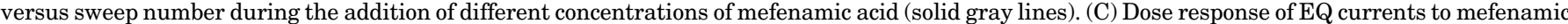

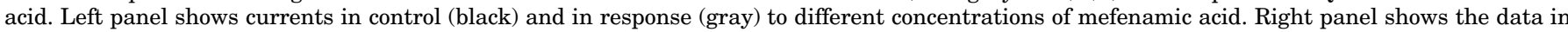

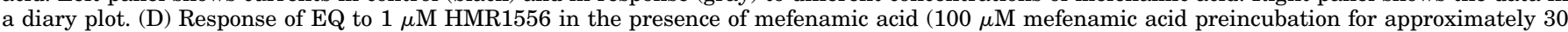

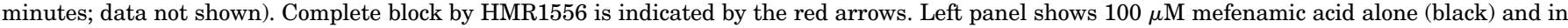

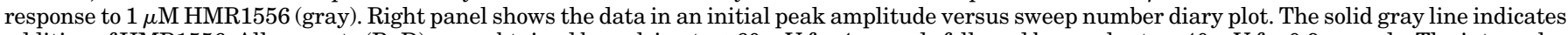

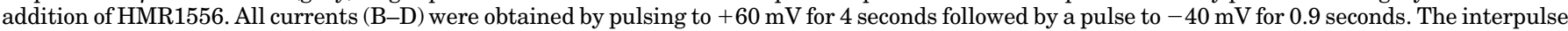
interval was 15 seconds. Holding potential was $-80 \mathrm{mV}$. Dotted baselines denote the zero-current level. 
A EQ

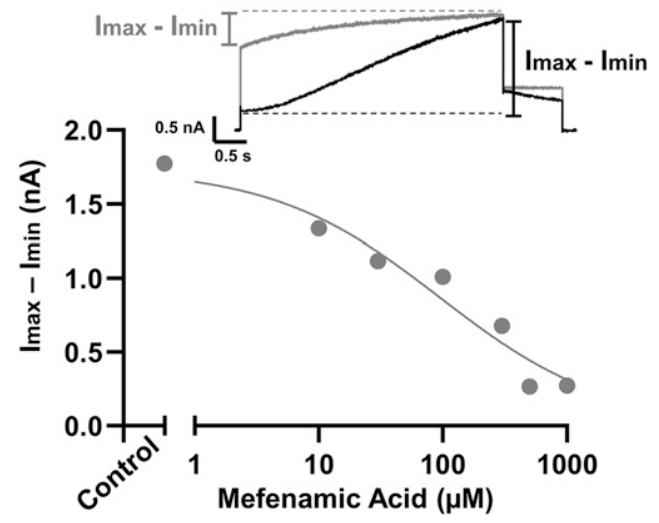

B

EQ

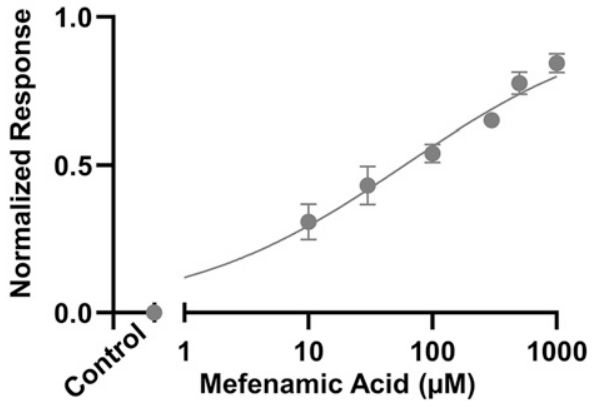

Fig. 2. Mefenamic acid dose-response curves for EQ. All currents used to calculate dose-response curves were obtained using the same protocol described in Fig. 1. (A) Representative dose-response calculation and curve. Upper panel shows representative $I_{\max }-I_{\min }$ measurements in the absence of mefenamic acid (black) and in the presence of $300 \mu \mathrm{M}$ mefenamic acid (gray). Lower panel shows $\mathrm{I}_{\max }-\mathrm{I}_{\min }$ versus log concentration of mefenamic acid (data from Fig. 1C). (B) Mean log concentration-response curve for $\mathrm{EQ}[n=$ $3-5$ at each concentration; $\mathrm{EC}_{50}=60 \mu \mathrm{M}$ (38, $89 \mu \mathrm{M} ; 95 \%$ confidence interval); $n^{H}=$ $0.49(0.39,0.60 ; 95 \%$ CI $)]$. Responses were normalized to the maximum peak to steady-state difference current in the absence of mefenamic acid and subtracted from 1.0 (see Materials and Methods). corresponding $\log _{10}$ concentration of mefenamic acid (Fig. 2B; Fig. 5D; Fig. 8B). Dose- and normalized-response curves were fit with a specific binding equation to obtain the $\mathrm{EC}_{50}$ and Hill coefficients $\left(n^{H}\right)$ as in Figs. 2, A and B, 5D, and 8B. The HMR1556 response diary plot in the presence of mefenamic acid (Fig. 1D, right panel) was obtained by plotting the initial peak current amplitude against the corresponding sweep number. All current-voltage (I-V) and conductance-voltage (G-V) plots in Figs. 3, 5, and 7 and Supplemental Fig. 1 were obtained from the normalized peak of the 4-second depolarizing pulses $\left(I / I_{\max }\right)$ and normalized peak of the initial tail current $\left(\mathrm{G} / \mathrm{G}_{\max }\right)$, respectively, and plotted against the corresponding voltage. G-V plots were fitted with a Boltzmann sigmoid equation to obtain the voltage at half-maximal activation $\left(\mathrm{V}_{1 / 2}\right)$ and slope $(k)$ values (Tables 1 and 2$)$. In the case of the mutant EQ $I_{K s}$, the change in $\mathrm{V}_{1 / 2}$ of activation $\left(\Delta \mathrm{V}_{1 / 2}=\mathrm{V}_{1 / 2}\right.$ in the presence of mefenamic acid $-\mathrm{V}_{1 / 2}$ control) was further determined (Fig. 7D; Table 2). In some cells, G-V relationships in the presence of mefenamic acid for wild-type (WT) EQ, K41R, and G40C were essentially linear, and consequently, the $\mathrm{V}_{1 / 2}$ of activation was read from the plots and included in the calculations of the mean values in Tables 1 and 2. All deactivation traces in Fig. 4, A and B were fitted with a single exponential equation to obtain the time constants of deactivation $\left(\tau_{\text {deact }}\right)$, which were plotted against the corresponding membrane potential (Fig. 4C).

\section{Modeling}

Markov modeling in Fig. 8 was carried out using the IonChannelLab software (Santiago-Castillo et al., 2010) incorporating Q-matrix solutions to the differential equations defining the kinetic behavior of rate transitions (Colquhoun and Hawkes, 1995). A balanced model (Zaydman et al., 2014) as revised by Westhoff et al. (2019) was used for simulations, using a 4:4 KCNQ1:KCNE1 stoichiometry. In this model, each voltage sensor (VS) is assumed to undergo two activating transitions, to an intermediate and then activated conformation. Pore subconductance opening can occur as soon as each VS is fully activated, and thus, pore opening does not require a concerted step after all four VS have reached fully activated conformations. For full model exposition and rates, see Westhoff et al. (2019). To simulate the action of mefenamic acid on $I_{K s}$ currents, the intrinsic rates of forward VS transitions at $0 \mathrm{mV}$, between resting and intermediate states $\left(\mathrm{k}_{\mathrm{RI}} 0\right)$ and between intermediate and activated states $\left(\mathrm{k}_{\mathrm{IA}} 0\right.$ ), were multiplied by the drug concentration ( $\mathrm{D}$, micromolar) or $\log _{10}[\mathrm{D}]$, respectively. This difference reflected the fact that an extreme hyperpolarization of the G-V relation and facilitation of initial steps of activation were caused by mefenamic acid at higher concentrations or rates of activation (Fig. 3), and thus, a greater acceleration of the first VS transition was required to simulate experimental data.

\section{Results}

Mefenamic Acid Increases EQ $I_{K s}$ Current Expressed in Mammalian Cells. Initially, to ensure that mefenamic acid (Fig. 1A) had no effect on the endogenous currents in ltkmouse fibroblast (LM) cells, different concentrations of mefenamic acid $(10,30,100,300,500 \mu \mathrm{M}$, and $1 \mathrm{mM})$ were perfused onto untransfected LM cells (Fig. 1B). At all concentrations of mefenamic acid, no changes were observed in the waveform (Fig. 1B, left panel) or $\mathrm{I}_{\max }-\mathrm{I}_{\min }$ (Fig. 1B, right panel) compared with untreated cells (control). A single concentration of mefenamic acid $(100 \mu \mathrm{M})$ has previously been shown to enhance $I_{K s}$ activity leading to a variable amount of instantaneous current and inhibition of tail current decay in Xenopus oocytes (Busch et al., 1994, 1997) and various mammalian cells (Unsöld et al., 2000; Magyar et al., 2006; Toyoda et al., 2006). The concentration dependence of this enhancement of $I_{K s}$ activity using different concentrations of mefenamic acid perfused onto LM cells transiently transfected with EQ is shown in Fig. 1C. With increasing concentrations of mefenamic acid, the control sigmoidal waveform (indicative of slow activation kinetics) was transformed into an almost linear waveform with significant instantaneous current (Fig. 1C, left panel). The corresponding difference current diary plot of increasing concentrations of mefenamic acid shows the gradual transformation to an instantaneous current over time (Fig. 1C, right panel). Consistent with previous findings, the decay in tail current seen in the control was also inhibited in a dose-dependent manner with increasing concentrations of mefenamic acid (Fig. 1C, left panel).

To confirm that this instantaneous current was produced by mefenamic acid enhancement of $I_{K s}$ activity specifically, we used the $I_{K s}$ blocker HMR1556 (Gögelein et al., 2000). In these experiments, LM cells transiently transfected with EQ were first preincubated in $100 \mu \mathrm{M}$ mefenamic acid for approximately 30 minutes (data not shown); then, when an $I_{K s^{-}}$ positive cell was identified, $1 \mu \mathrm{M}$ HMR1556 was applied to the bath (Fig. 1D). As is evident in the representative traces (Fig. 1D, left panel) and diary plot (Fig. 1D, right panel), following HMR1556 treatment, the instantaneous current amplitude decreased over time to that of endogenous current amplitudes, suggesting that the instantaneous current was, in fact, flowing through $I_{K s}$ channels.

Mefenamic Acid Dose-Response Curves for EQ $\boldsymbol{I}_{\boldsymbol{K} \boldsymbol{s}}$. Increasing concentrations of mefenamic acid gradually 

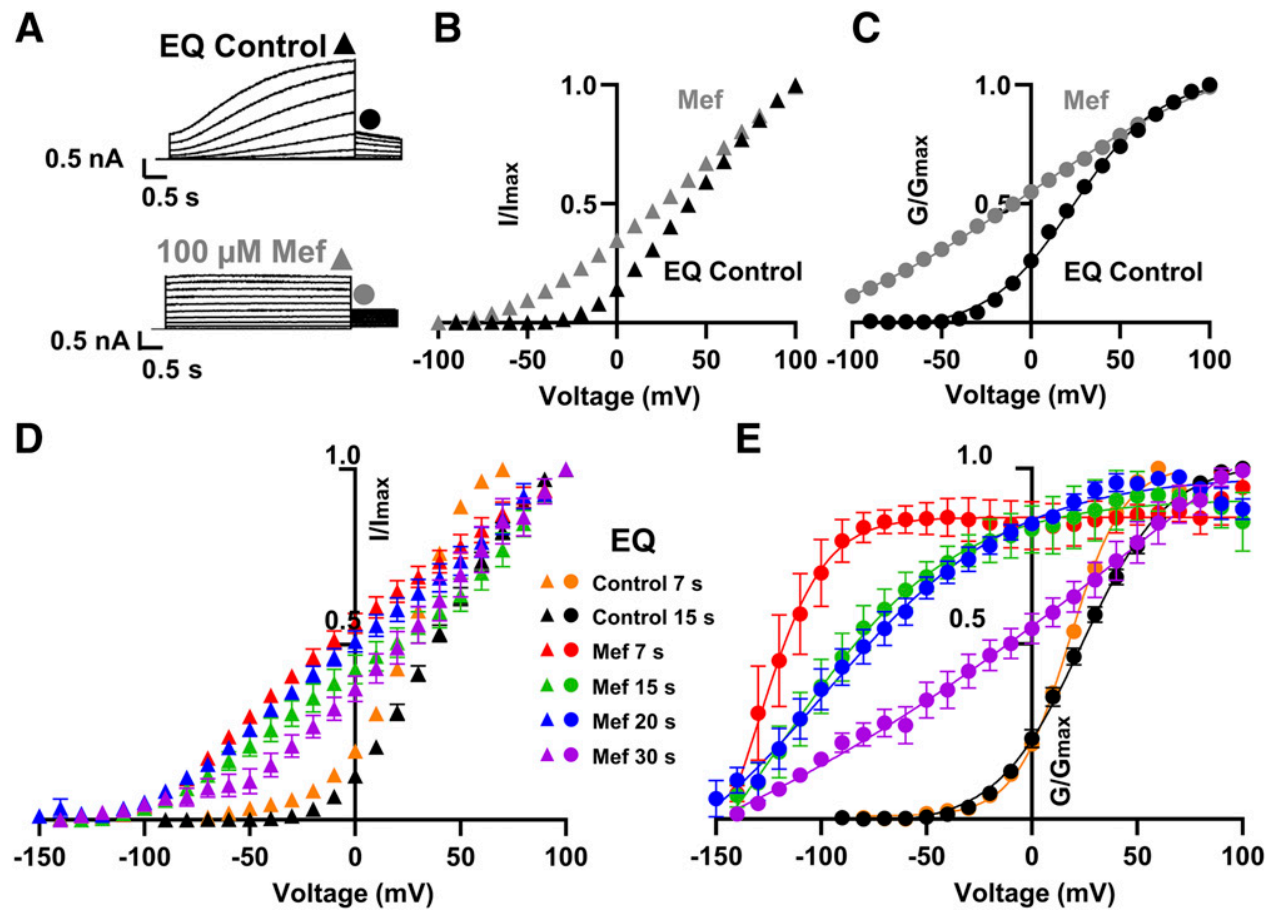

Fig. 3. Mefenamic acid rate-dependently hyperpolarizes EQ I-V and G-V relationships. Currents were obtained using a 4-second step protocol with pulses from -150 to $+100 \mathrm{mV}$, followed by a repolarizing step to $-40 \mathrm{mV}$ for 1 second. (A) Currents are shown for EQ in the absence (control; interpulse interval was 15 seconds; upper panel) and presence of $100 \mu \mathrm{M}$ mefenamic acid (Mef; interpulse interval was 30 seconds; lower panel). (B) I-V plots of current at the end of the 4-second depolarizing pulses in control (black triangles) and mefenamic acid (gray triangles); data from (A). (C) G-V relationships obtained from peak initial tail currents in control $\left(\mathrm{V}_{1 / 2}=24.0 \mathrm{mV} ; k=24.0 \mathrm{mV}\right.$; black circles) and mefenamic acid $\left(\mathrm{V}_{1 / 2}=-2.4 \mathrm{mV} ; k=65.4 \mathrm{mV} ;\right.$ gray circles); data from (A). (D and E) Effect of different interpulse intervals on I-V (triangles) and G-V (circles) plots in control and mefenamic acid. Intervals were 7 ( $n=1$; orange) and 15 seconds ( $n=4$; black) in control, and 7 (red), 15 (green), 20 (blue), or 30 seconds (purple) in mefenamic acid ( $n=4-6$ ). For G-V plots, Boltzmann fits were for $7\left(\mathrm{~V}_{1 / 2}=17.8 \mathrm{mV} ; k=12.9 \mathrm{mV}\right)$ and 15 seconds $\left(\mathrm{V}_{1 / 2}=23.9 \mathrm{mV} ; k=20.4 \mathrm{mV}\right)$ in control, and for $7-\left(\mathrm{V}_{1 / 2}=-108 \mathrm{mV} ; k=6.9\right.$ $\mathrm{mV}), 15-\left(\mathrm{V}_{1 / 2}=-86.5 \mathrm{mV} ; k=23.2 \mathrm{mV}\right), 20-\left(\mathrm{V}_{1 / 2}=-80.5 \mathrm{mV} ; k=53.3 \mathrm{mV}\right)$, and 30 -second intervals $\left(\mathrm{V}_{1 / 2}=-13.1 \mathrm{mV} ; k=57.2 \mathrm{mV}\right)$ in mefenamic acid (see Table 1). $\mathrm{V}_{1 / 2}$ values for mefenamic acid compared with control were significantly different at all intervals (Table 1).

transformed the sigmoidal activation waveform of EQ $I_{K s}$ into an almost linear waveform, and this change was quantified by measuring peak to steady-state difference currents (Fig. 2A, upper panel; see Materials and Methods). More specifically, difference currents were calculated by subtracting the initial amplitude of the activating current $\left(\mathrm{I}_{\text {min }}\right)$ from the peak amplitude of the activating current $\left(\mathrm{I}_{\max }\right)$ and plotting the result against the corresponding $\log$ concentration of mefenamic acid (Fig. 2A). Normalized-response relationships for EQ were obtained by transformation of $I_{\max }-I_{\min }$ data (see Materials and Methods) and fit with a specific binding equation (Fig. 2B). The $\mathrm{EC}_{50}$ and Hill coefficient $\left(n^{H}\right)$ for mefenamic acid were $60 \mu \mathrm{M}$ and 0.49 , respectively. To ensure consistent results and to allow for comparison with previous literature, all subsequent experiments to characterize the gating properties, subunit stoichiometry, and specific regulatory residues were conducted using $100 \mu \mathrm{M}$ mefenamic acid, unless otherwise stated.

Mefenamic Acid Rate-Dependently Hyperpolarizes EQ $I_{K s}$ I-V and G-V Relationships. To investigate what happens to EQ $I_{K s} \mathrm{I}-\mathrm{V}$ and $\mathrm{G}-\mathrm{V}$ relationships following treatment with $100 \mu \mathrm{M}$ mefenamic acid, a 4-second activation protocol with varying interpulse intervals was used (Fig. 3). Fig. 3A shows representative waveforms of EQ $I_{K s}$ in both the absence (control; upper panel) and presence of mefenamic acid (lower panel), and shows the characteristic delay of current activation in control and the appearance of an instantaneous current with mefenamic acid treatment. The corresponding
I-V (Fig. 3B) and G-V relationships (Fig. 3C) were obtained by plotting the normalized peak amplitudes at the end of the 4-second depolarizing pulses ( $\mathrm{I} / \mathrm{I}_{\max }$ ) or the normalized peak of the initial tail current $\left(\mathrm{G} / \mathrm{G}_{\max }\right)$, respectively, against the corresponding voltage. During exposure to mefenamic acid, the I-V relationship became more linear and hyperpolarized (Fig. 3B). The G-V relationship following treatment with mefenamic acid was also hyperpolarized (control: $\mathrm{V}_{1 / 2}=24.0$ $\mathrm{mV}, k=24.0 \mathrm{mV}$; mefenamic acid: $\mathrm{V}_{1 / 2}=-2.4 \mathrm{mV}, k=65.4$ $\mathrm{mV}$; Fig. 3C).

Visually, all I-V relationships at different interpulse intervals $(30,20,15$, and 7 seconds) in the presence of mefenamic acid appeared almost linear and more hyperpolarized (Fig. 3D). The degree of hyperpolarization was graded with increasingly shorter intervals, and I-V relationships for interpulse intervals of 7 and 30 seconds were the most and least hyperpolarized, respectively. In contrast, altering the interpulse interval dramatically affected the G-V relationships of mefenamic acid-treated EQ (Fig. 3E). The degree of hyperpolarization of the G-V plots and $V_{1 / 2}$ of activation was also greater with shorter interpulse intervals. The G-V relationship and $\mathrm{V}_{1 / 2}$ for an interpulse interval of 7 seconds $(-108 \mathrm{mV})$ was the most hyperpolarized, followed by the G-V relationships and $\mathrm{V}_{1 / 2}$ values at interpulse intervals of $15(-86.5 \mathrm{mV}) ; 20(-80.5 \mathrm{mV})$; and, finally, 30 seconds $(-13.1 \mathrm{mV})$, which was the least hyperpolarized (Table 1). The $\mathrm{V}_{1 / 2}$ of activation at all tested interpulse intervals (30, 20,15 , and 7 seconds) in the presence of mefenamic acid was 

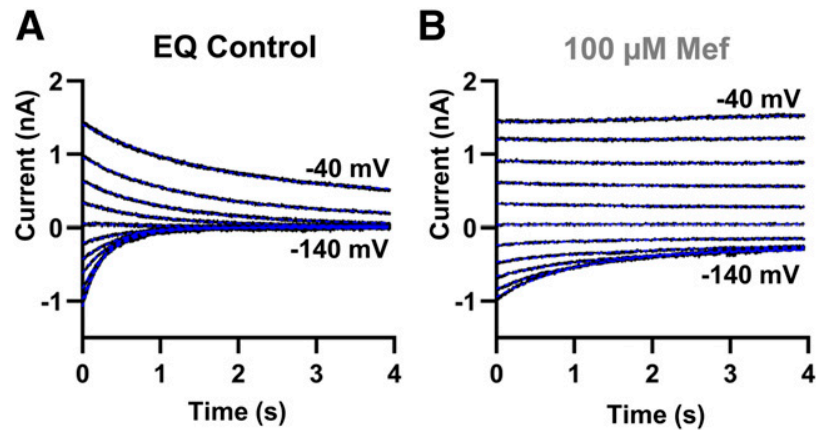

C

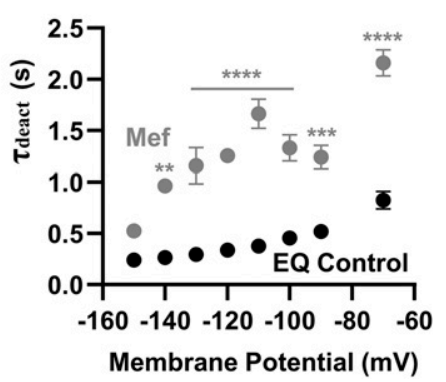

Fig. 4. Mefenamic acid (Mef) slows EQ deactivation. Tail currents were obtained by pulsing to $+60 \mathrm{mV}$ for 4 seconds to activate $I_{K s}$ current, followed by a 4-second pulse to a range of potentials from -40 to $-150 \mathrm{mV}$ in $10-\mathrm{mV}$ steps. Holding potential was $-90 \mathrm{mV}$. (A) Representative tail currents (black) fit with a single exponential curve (blue lines) for EQ $I_{K s}$ in the absence of mefenamic acid. (B) Representative tail currents (black) fit with a single exponential curve (blue lines) for EQ $I_{K s}$ in the presence of $100 \mu \mathrm{M}$ mefenamic acid. (C) Time constants of deactivation $\left(\tau_{\text {deact }}\right)$ versus different membrane potentials for $\mathrm{EQ} I_{K s}$ in the absence $(n=3$, black circles) and presence of $100 \mu \mathrm{M}$ mefenamic acid $(n=5$, gray circles). $* * P=0.0089$; $* * * P=0.0003$; and $* * * * P<0.0001$

significantly different from control (Table 1). The slope of the G-V relationship was significantly decreased when the interpulse interval was either $20(53.3 \mathrm{mV}$; $P=0.0045)$ or 30 seconds $(57.2 \mathrm{mV} ; P=0.0035)$ in the presence of mefenamic acid when compared with control $(20.4 \mathrm{mV}$ ) (Table 1). The slope of the G-V relationship, however, was not significantly different when the interpulse interval was $15(23.2 \mathrm{mV})$ or 7 seconds $(6.9 \mathrm{mV})$ in the presence of mefenamic acid when compared with control (Table 1).
Altering the interpulse interval of the activation protocol to 7 seconds, however, did not significantly affect the control (in the absence of mefenamic acid) I-V and G-V relationships (Fig. 3, D and E), although the G-V relationship did become steeper. Overall, these results show that mefenamic acid hyperpolarizes the I-V and G-V relationships of EQ $I_{K s}$ in a rate-dependent fashion.

Mefenamic Acid Slows EQ $I_{K s}$ Deactivation. To investigate changes in rates of $\mathrm{EQ}$ deactivation following treatment with $100 \mu \mathrm{M}$ mefenamic acid, tail currents were

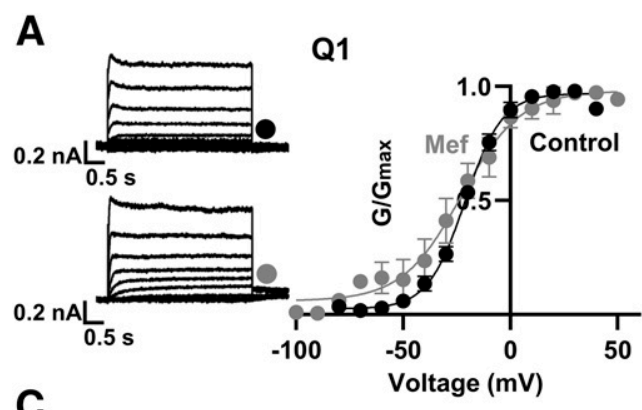

B
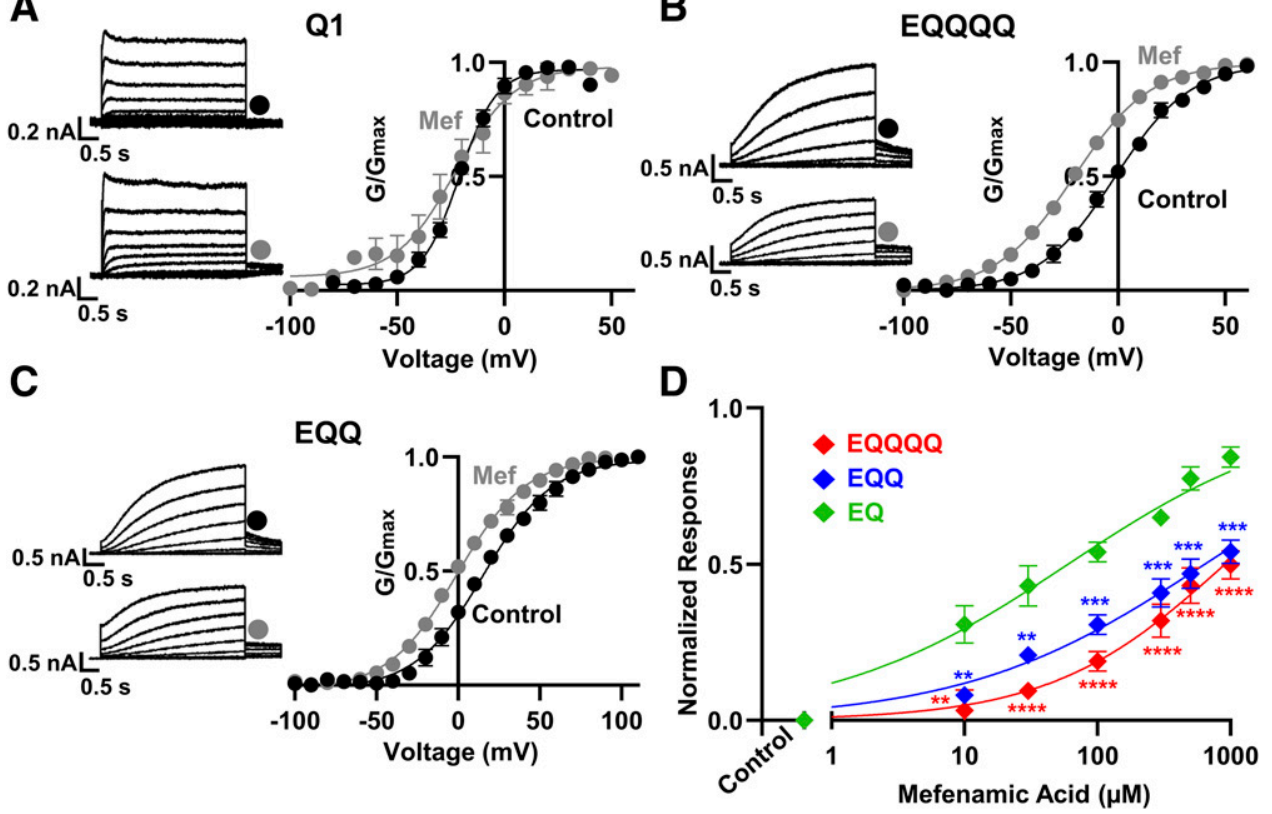

Fig. 5. Effect of $I_{K s}$ stoichiometry on response to $100 \mu \mathrm{M}$ mefenamic acid. The currents and conductance-voltage plots (A-C) were obtained using the protocols described in Fig. 3 with a 15 second interpulse interval. For each stoichiometry (see Materials and Methods), representative currents are shown in the absence of (control; upper left panel) and in the presence of $100 \mu \mathrm{M}$ mefenamic acid (lower left panel). Right panels show the corresponding G-V plots in control (black circles) and presence of mefenamic acid (gray circles). Boltzmann fits were: (A) for Q1 in control $\left(n=5\right.$ ): $\mathrm{V}_{1 / 2}=-20.5 \mathrm{mV}, k=8.8$ $\mathrm{mV}$; and, in mefenamic acid $(n=4): \mathrm{V}_{1 / 2}=-25.6 \mathrm{mV}, k=14.9 \mathrm{mV}$; (B) for EQQQQ $I_{K_{s}}$ in control $(n=4): \mathrm{V}_{1 / 2}=-1.5 \mathrm{mV}, k=18.1 \mathrm{mV}$; and, in mefenamic acid $(n=4): \mathrm{V}_{1 / 2}=-20.8 \mathrm{mV}, k=18.3 \mathrm{mV}$; (C) for $\mathrm{EQQ} I_{K s}$ in control $(n=5): \mathrm{V}_{1 / 2}=15.4 \mathrm{mV}, k=20.6 \mathrm{mV}$; and in mefenamic acid $(n=4) \mathrm{V}_{1 / 2}=-1.6 \mathrm{mV}$, $k=20.3 \mathrm{mV}$ (See Table 2). (D) Normalized log concentration-response relationships for EQ, EQQ and EQQQQ $I_{K s}$ were obtained using the analysis method described in Fig. 2. For EQ $I_{K s}: \mathrm{EC}_{50}=60[38,89 ; 95 \% \mathrm{CI}] \mu \mathrm{M}, n^{H}=0.49\left[0.39,0.60 ; 95 \% \mathrm{CI}\right.$, green diamonds, data from Fig. 2B; for EQQ $I_{K s}$ : $\mathrm{EC}_{50}=615[422,955 ; 95 \% \mathrm{CI}] \mu \mathrm{M}, n^{H}=0.47[0.37,0.60 ; 95 \% \mathrm{CI}]$, blue diamonds; and for EQQQQ $I_{K s}: \mathrm{EC}_{50}=902[663,1383 ; 95 \% \mathrm{CI}] \mu \mathrm{M}, n^{H}=0.66[0.49$, $0.87 ; 95 \% \mathrm{CI}]$, red diamonds. For each construct, $n=3-5$ at each concentration. ${ }^{* *}, * * *$ and $* * * *$ denotes a significantly different response when compared with EQ $I_{K s}$ and, where $P<0.05, P<0.0005$ and $P<0.0001$, respectively. 


\section{TABLE 1}

$\mathrm{V}_{1 / 2}$ of activation (millivolts) and slope value ( $k$-factor, millivolts) in the absence and presence of $100 \mu \mathrm{M}$ mefenamic acid for EQ $I_{K s}$ at different interpulse intervals

The \pm denotes S.D., with the $P$ value indicating statistical difference in $V_{1 / 2}$ compared with control as determined using a one-way ANOVA and Bonferron multiple comparisons test.

\begin{tabular}{lcccc}
\hline & $\mathrm{V}_{1 / 2}$ & $k$-Factor & $n$ & $P$ Value \\
\hline $\begin{array}{c}\text { EQ control: interpulse } \\
\text { interval 15 s }\end{array}$ & $23.9 \pm 3.7$ & $20.4 \pm 2.9$ & 4 & \\
$\begin{array}{c}\mathrm{EQ}+\text { mefenamic acid: } \\
\text { interpulse interval 7 s }\end{array}$ & $-108 \pm 9.0$ & $6.9 \pm 7.7$ & 6 & $<0.0001$ \\
$\begin{array}{c}\text { EQ + mefenamic acid: } \\
\text { interpulse interval 15 s }\end{array}$ & $-86.5 \pm 14.8$ & $23.2 \pm 11.2$ & 4 & $<0.0001$ \\
$\begin{array}{c}\text { EQ + mefenamic acid: } \\
\text { interpulse interval 20 s }\end{array}$ & $-80.5 \pm 18.2$ & $53.3 \pm 15.3$ & 5 & $<0.0001$ \\
$\begin{array}{c}\text { EQ + mefenamic acid: } \\
\text { interpulse interval 30 s }\end{array}$ & $-13.1 \pm 14.9$ & $57.2 \pm 15.4$ & 6 & 0.0057 \\
\hline
\end{tabular}

obtained in the absence (Fig. 4A) and presence of mefenamic acid (Fig. 4B) and fit with single exponential decay curves. Deactivation time constants were obtained from these fits and plotted against the membrane potential (Fig. 4C). The $\mathrm{K}^{+}$ reversal potential was found to be approximately $-80 \mathrm{mV}$, and therefore, the rate of deactivation at $-80 \mathrm{mV}$ was omitted. Treatment with mefenamic acid significantly decreased the rate of deactivation at -70 to $-140 \mathrm{mV}$.

Effect of $I_{K s}$ Stoichiometry on Response to $100 \mu \mathrm{M}$ Mefenamic Acid. To investigate whether the effect of mefenamic acid on $I_{K s}$ dose response and G-V relationships was dependent on the E1:Q1 stoichiometry, mammalian cells (LM and tsA201 cells) were transiently transfected with EQ $I_{K s}, \mathrm{EQQ} I_{K s}, \mathrm{EQQQQ} I_{K s}$, or KCNQ1 alone (Fig. 5). These $I_{K s}$ constructs fix the ratio of E1:Q1 to $4: 4,2: 4$, or $1: 4$ through linking the $\mathrm{C}$ terminus of $\mathrm{KCNE} 1$ to the $\mathrm{N}$ terminus of one, two, or four KCNQ1 sequences, respectively.

No change in the KCNQ1 waveform was seen following treatment with $100 \mu \mathrm{M}$ mefenamic acid (control: Fig. 5A, upperleft panel; mefenamic acid: Fig. 5A, lower-left panel). Mefenamic acid also did not significantly shift the $\mathrm{V}_{1 / 2}$ of activation or slope of the G-V relationship (Table 2), supporting the conclusion that mefenamic acid has no effect on KCNQ1 alone.
When one KCNE1 subunit was present (EQQQQ $I_{K s}$ ), the instantaneous current characteristic of mefenamic acid's effect on EQ $I_{K s}$ no longer occurred (Fig. 5B, left panel). The EQQQQ $I_{K s}$ waveform was sigmoidal in both the absence (Fig. 5A, upper-left panel) and presence of $100 \mu \mathrm{M}$ mefenamic acid (Fig. 5A, lower-left panel). Despite mefenamic acid not having a dramatic transformative effect on the activation waveform of EQQQQ, inhibition of EQQQQ tail current decay following mefenamic acid treatment still occurred. A significant leftward shift in the $\mathrm{V}_{1 / 2}$ of activation also occurred (control: $-1.5 \mathrm{mV}$; mefenamic acid: $-20.8 \mathrm{mV} ; P=0.0298$; Table 2). There were no significant changes in the slope of the G-V relationships.

Similar to EQQQQ $I_{K s}$, there was no dramatic transformative effect on the EQQ $I_{K s}$ activation waveform following mefenamic acid treatment (Fig. 5C, left panel). Inhibition of EQQ $I_{K s}$ tail current decay, however, still occurred. A significant leftward shift in the $\mathrm{V}_{1 / 2}$ of activation also occurred (control: $15.4 \mathrm{mV}$; mefenamic acid: $-1.6 \mathrm{mV} ; P=0.0472$; Table 2). Again, the slope of the G-V relationships did not change. Overall, the leftward shift in the $\mathrm{V}_{1 / 2}$ of activation $\left(\Delta \mathrm{V}_{1 / 2}\right)$ for EQQQQ $(-19.3 \mathrm{mV})$ and EQQ $(-17.1 \mathrm{mV})$ was less dramatic than the $\Delta \mathrm{V}_{1 / 2}$ of activation for $\mathrm{EQ}(-110 \mathrm{mV}$; Table 2). The normalized responses of the different $I_{K s}$ stoichiometries (normalized difference currents) to different concentrations of mefenamic acid are plotted in Fig. 5D. At all concentrations, the normalized responses of EQQQQ $I_{K s}$ and EQQ $I_{K s}$ were significantly reduced compared with the response of EQ $I_{K s}$. The $\mathrm{EC}_{50}$ and $n^{H}$ were $902 \mu \mathrm{M}$ and 0.66 for EQQQQ $I_{K s}, 615 \mu \mathrm{M}$ and 0.47 for EQQ $I_{K s}$, and $60 \mu \mathrm{M}$ and 0.49 for EQ $I_{K s}$, respectively. Since no change in the KCNQ1 waveform or significant shift in the $\mathrm{V}_{1 / 2}$ was seen following mefenamic acid treatment (Fig. 5A), these data were not included in the log concentration-normalized response plot in Fig. 5D.

Mefenamic Acid Effects at the Single-Channel Level. To determine if the enhancement of $I_{K s}$ current upon mefenamic acid treatment was simply a result of an increase in open probability or there were additional effects on conductance, we made single-channel recordings of $4: 4 I_{K s}$ stoichiometry (EQ) in the presence of the drug. Figure 6A shows

TABLE 2

$\mathrm{V}_{1 / 2}$ of activation (millivolts) and slope value ( $k$-factor, millivolts) in the absence and presence of mefenamic acid for mutant EQ $I_{K s}$ and different stoichiometrically saturated WT $I_{K s}$

The \pm denotes S.D., with the $P$ value indicating statistical difference in $\mathrm{V}_{1 / 2}$ compared with control as determined using a one-way ANOVA and Bonferroni multiple comparisons test.

\begin{tabular}{|c|c|c|c|c|c|c|c|c|}
\hline & \multicolumn{3}{|c|}{ Control } & \multicolumn{3}{|c|}{$100 \mu \mathrm{M}$ or $1 \mathrm{mM}$ Mefenamic $\mathrm{Acid}^{a}$} & $\Delta \mathrm{V}_{1 / 2}$ & $P$ Value \\
\hline $\mathrm{EQ}$ & $23.9 \pm 3.7$ & $20.4 \pm 2.9$ & 4 & $-86.5 \pm 14.8$ & $23.2 \pm 11.2$ & 4 & -110 & $<0.0001$ \\
\hline EQQQQ & $-1.5 \pm 5.0$ & $18.1 \pm 1.1$ & 4 & $-20.8 \pm 3.7$ & $18.3 \pm 3.0$ & 4 & -19.3 & 0.0298 \\
\hline Q1 & $-20.5 \pm 1.4$ & $8.8 \pm 2.9$ & 5 & $-25.6 \pm 12.9$ & $14.9 \pm 4.7$ & 4 & -5.1 & NS \\
\hline E43C & $70.8 \pm 3.5$ & $27.0 \pm 5.5$ & 4 & $46.4 \pm 8.4$ & $29.1 \pm 3.4$ & 4 & -24.4 & 0.0228 \\
\hline L42C & $68.9 \pm 2.6$ & $21.5 \pm 6.4$ & 3 & $31.8 \pm 0.6$ & $14.8 \pm 7.4$ & 3 & -37.2 & 0.0011 \\
\hline \multirow[t]{2}{*}{$\mathrm{K} 41 \mathrm{E}$} & $76.5 \pm 5.5$ & $24.2 \pm 0.8$ & 4 & $102 \pm 7.5$ & $25.0 \pm 2.0$ & 4 & 24.9 & 0.0189 \\
\hline & & & & $115 \pm 8.0$ & $25.6 \pm 1.7$ & 3 & 38.1 & 0.0003 \\
\hline $\mathrm{K} 41 \mathrm{R}$ & $72.7 \pm 5.8$ & $20.8 \pm 3.2$ & 3 & $26.6 \pm 21.1$ & $49.3 \pm 19.4$ & 4 & -46.2 & $<0.0001$ \\
\hline G40C & $38.2 \pm 11.8$ & $20.0 \pm 2.2$ & 4 & $-37.9 \pm 23.5$ & $\sim 40.3$ & 3 & -76.1 & $<0.0001$ \\
\hline
\end{tabular}

NS, not significant.

${ }^{a}$ Mefenamic acid dose was either $100 \mu \mathrm{M}$ (where applicable, upper row values) or $1 \mathrm{mM}$ (where applicable, lower row values). Given the dramatic effect mefenamic acid has on the G-V relationship for some constructs, Boltzmann curves could not be properly fit in some cases. 
three representative active traces of control EQ and three in the presence of $100 \mu \mathrm{M}$ mefenamic acid. The voltage protocol was analogous to the whole-cell experiments in that a 4-second depolarization was given, with a 0.75 -second repolarization period at $-40 \mathrm{mV}$, applied every 10 seconds.

It is clear from these records that channels opened very early upon depolarization in the presence of mefenamic acid (Fig. 6A, bottom-three sweeps), and that channel openings were persistent during the repolarization step to $-40 \mathrm{mV}$. Thus, first latency was shortened and deactivation slowed in the presence of mefenamic acid, as would be expected based on the whole-cell recordings. Note that, during exposure to mefenamic acid, channels were not already activated when initially depolarized, as might have been expected if the effects of mefenamic acid were simply related to a failure of deactivation between pulses. It is clear that channels activate de novo with each pulse. This is confirmed in the ensemble averages of 18 active sweeps of control and 18 sweeps from the same recording taken approximately 23 minutes after mefenamic acid exposure (Fig. 6B), where channel activity was seen earlier during the depolarization in the presence of mefenamic acid (red tracing) than in control (blue tracing), and tail currents persisted to the end of the recording period. The all-points histograms (Fig. 6C) comparing events in the three control and three mefenamic acid sweeps shown in Fig. 6A also show a reduction in the number of closed events, which is indicative of increased channel activity and decreased latency in the presence of mefenamic acid. In addition, the peak open amplitude around $0.4 \mathrm{pA}$ is maintained but shows more events due to prolonged opening bursts seen in the presence of mefenamic acid. Maximum channel conductance was not increased by mefenamic acid; rather, simply more open events were seen at the same levels present in control.

Another important observation from the single-channel experiments using the cell-attached configuration was the delay in action of mefenamic acid. In whole-cell experiments, mefenamic acid effects were observed within approximately 0.5-1 minute of bath application, whereas when the extracellular domain of $I_{K s}$ was shielded by the recording electrode in the cell-attached configuration, the effect took up to 20 minutes to become obvious. This supports previous research pointing to an extracellular binding site for the drug (Abitbol et al., 1999). When mefenamic acid was included in the patch pipette, the effect on open probability was fairly immediate (data not shown).

Mapping the Mefenamic Acid Regulatory Sites on KCNE1. Since our data indicate that mefenamic acid has minimal effect on KCNQ1 alone, and the effect of mefenamic acid is dependent upon channel stoichiometry, we further examined potential mefenamic acid regulatory sites on KCNE1. The binding site for mefenamic acid has previously been suggested to lie between residues 39 and 43 on KCNE1 (Abitbol et al., 1999); however, the importance of each residue to mefenamic acid's subsequent effect on $I_{K s}$ has not previously been characterized. Using mutational analysis, we therefore characterized how singularly mutating residues in this region would affect mefenamic acid's ability to alter the waveform and G-V relationship of EQ $I_{K s}$.

Similar to WT EQ $I_{K s}$, all mutant EQ $I_{K s}$ showed the characteristic delay of current activation in the absence of mefenamic acid (L42C, Fig. 7A; K41C, Fig. 7B; K41R, Fig. 7C; G40C, Supplemental Fig. 1A; current data not shown for K41E and E43C). Additionally, the characteristic appearance of instantaneous current and inhibition of tail current decay induced by mefenamic acid on WT EQ $I_{K s}$ was preserved in the G40C (Supplemental Fig. 1B), L42C (Fig. 7A), and E43C (data not shown) EQ $I_{K s}$ mutants. When an interpulse interval of 15 seconds was used, mefenamic acid also significantly altered the shape of the G-V relationship (Supplemental Fig. 1C) and left shifted the $\mathrm{V}_{1 / 2}$ of activation for $\mathrm{G} 40 \mathrm{C}\left(\Delta \mathrm{V}_{1 / 2}:-76.1 \mathrm{mV}\right)$ (Fig. 7D; Table 2). This effect on the G-V relationship and $\mathrm{V}_{1 / 2}$ of G40C was also dependent on the interpulse interval-with the shortest interpulse interval (interpulse intervals examined include 7,15 , and 30 seconds) producing the most dramatically altered G-V relationship (Supplemental Fig. $1 \mathrm{C})$ and visually the most leftward shift in $\mathrm{V}_{1 / 2}$. In contrast,

A

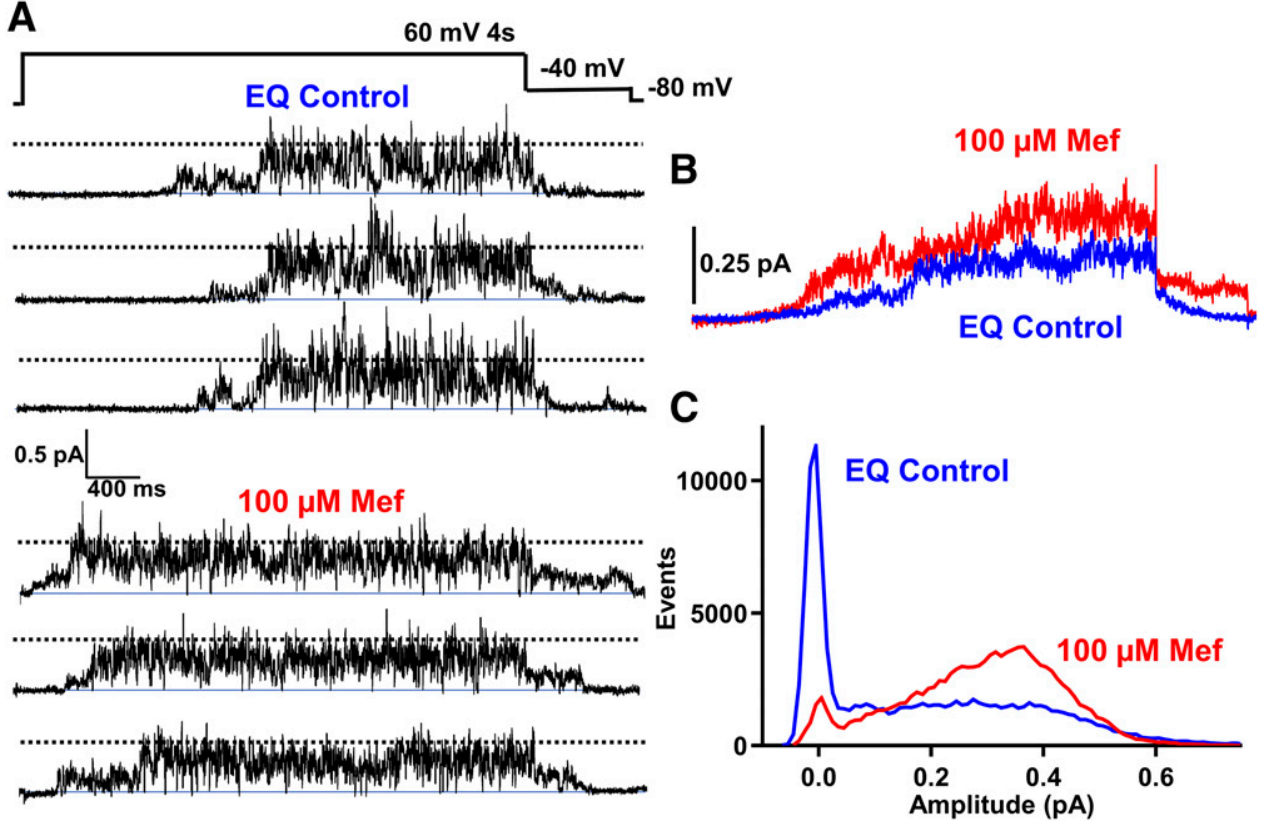

Fig. 6. Mefenamic acid (Mef) increases the open probability of $I_{K s}$ and not the conductance. (A) Single-channel currents were obtained using the protocol shown. The sweep-to-sweep interval was 10 seconds. Representative single-channel traces are shown for control EQ (top-three sweeps) and from the same cell after approximately 23 minutes of $100 \mu \mathrm{M}$ mefenamic acid exposure (bottom-three sweeps). Black dotted lines indicate the 0.5-pA level. Blue lines indicate the zerocurrent level. (B) Ensemble averages of 18 active sweeps each of $\mathrm{EQ}$ (blue) and in the presence of $100 \mu \mathrm{M}$ mefenamic acid (red). (C) All-points histograms of the three control EQ sweeps (blue) and the three sweeps in the presence of mefenamic acid (red) shown in (A). 
the effect mefenamic acid had on the G-V relationships of L42C and E43C was not as dramatic (L42C: Fig. 7A, right panel; data not shown for E43C). When an interpulse interval of 15 seconds was used, the shape of the G-V relationship in both the absence and presence of mefenamic acid was sigmoidal for $\mathrm{L} 42 \mathrm{C}$ and $\mathrm{E} 43 \mathrm{C}$. In addition, the slope of the G-V relationship was not significantly altered following mefenamic acid treatment; however, a significant hyperpolarizing shift in the $\mathrm{V}_{1 / 2}$ of activation occurred $\left(\Delta \mathrm{V}_{1 / 2}\right.$ for $\mathrm{L} 42 \mathrm{C}$ : $-37.2 \mathrm{mV} ; \Delta \mathrm{V}_{1 / 2}$ for E43C: $-24.4 \mathrm{mV}$ ) (Fig. 7D; Table 2) for both mutants. Therefore, mutating residues L42, E43, and especially G40 on KCNE1 only minimally reduced mefenamic acid's effect on EQ $I_{K s}$.

Mutations made at $\mathrm{K} 41$ on $\mathrm{KCNE} 1$ had a different result. Unlike WT EQ $I_{K s}$, after treatment with $100 \mu \mathrm{M}$ mefenamic acid, the waveform of $\mathrm{K} 41 \mathrm{C}$ still showed delayed current activation and WT tail current decay (Fig. 7B, lower-left panel). Moreover, there was also no change in the slope and shape of the $\mathrm{G}-\mathrm{V}$ relationship (Fig. 7B, right panel) or significant shift in the $\mathrm{V}_{1 / 2}$ of activation (Fig. 7D; Table 2). Even in the presence of $1 \mathrm{mM}$ mefenamic acid, there was still no effect on the slope and shape of the G-V relationship (Fig. 7B, right panel) or significant shift in the $\mathrm{V}_{1 / 2}$ of activation when compared with control (Fig. 7D; Table 2). Therefore, mutating residue K41 drastically reduced mefenamic acid's effect on EQ $I_{K s}$. We hypothesized that neutralization of the positively charged K41 was responsible for the loss of efficacy of mefenamic acid. In support of this idea, similar to K41C, after treatment with $100 \mu \mathrm{M}$ mefenamic acid, the waveform of K41E still showed WT-like delayed current activation and tail current decay (data not shown). Although there was once again no change in the slope and shape of the G-V relationship (data not shown), a significant right shift in the $\mathrm{V}_{1 / 2}$ of activation compared with control was seen with $100 \mu \mathrm{M}$ mefenamic acid $\left(\Delta \mathrm{V}_{1 / 2}\right.$ for $\mathrm{K} 41 \mathrm{E}$ with $100 \mu \mathrm{M}:+24.9$ $\mathrm{mV}$ ) (Fig. 7D; Table 2). Even in the presence of $1 \mathrm{mM}$ mefenamic acid, there was no effect on the slope and shape of the G-V relationship but still a right shift in $\mathrm{V}_{1 / 2}$ of activation compared with control $\left(\Delta \mathrm{V}_{1 / 2}\right.$ for $\mathrm{K} 41 \mathrm{E}$ with $1 \mathrm{mM}$ : $+38.1 \mathrm{mV}$ ) (Fig. 7D; Table 2).

In contrast, much like G40C, treatment with $100 \mu \mathrm{M}$ mefenamic acid transformed the slowly activating waveform of $\mathrm{K} 41 \mathrm{R}$ (Fig. 7C, upper-left panel) into one which has an instantaneous current and inhibited tail current decay (Fig. 7C, lower-left panel) characteristic of mefenamic acid's effect on WT EQ $I_{K s}$. Mefenamic acid also significantly altered the shape of the K41R G-V relationship (Fig. 7C, right panel) when an interpulse interval of 15 seconds was used. This effect on the shape of the K41R G-V relationship, however, was visually less dramatic than that of G40C and WT EQ $I_{K s}$ (WT EQ $I_{K s}$ G-V plots in control and $100 \mu \mathrm{M}$ mefenamic acid, both with an interpulse interval of 15 seconds, are overlaid on the Fig. 7C right panel for comparison; $\Delta \mathrm{V}_{1 / 2}:-110 \mathrm{mV}$ ). Despite this, the effect mefenamic acid had on the G-V relationship of $\mathrm{K} 41 \mathrm{R}$ was also dependent on the interpulse interval (data not shown). A shorter interpulse interval of 7 seconds produced a more dramatically altered G-V relationship and more leftward shift in $V_{1 / 2}$ than when the interpulse interval was


Fig. 7. Mapping the mefenamic acid (Mef) regulatory sites on KCNE1. The currents and G-V plots (A-C) were obtained using the protocols described in Fig 3. The interpulse interval was 15 seconds. For each EQ $I_{K s}$ mutant, representative currents are shown in the absence (control; upper-left panel) and presence of $100 \mu \mathrm{M}$ mefenamic acid (lower-left panel). Right panels show the corresponding G-V plots in control (black circles) and presence of $100 \mu \mathrm{M}$ (gray circles) and $1 \mathrm{mM}$ (purple circles) mefenamic acid. WT EQ $I_{K s}$ in control (blue triangles) and in the presence of $100 \mu \mathrm{M}$ mefenamic acid (red triangles), both with a 15-second interpulse interval, is overlaid in (C) (data from Fig $3 \mathrm{E}$ ). Boltzmann fits were as follows: for $\mathrm{L} 42 \mathrm{C}, \mathrm{V}_{1 / 2}=68.9 \mathrm{mV}$ and $k=$ $21.5 \mathrm{mV}$ in control $(n=3)$, and $\mathrm{V}_{1 / 2}=31.8 \mathrm{mV}$ and $k=14.8 \mathrm{mV}$ in $100 \mu \mathrm{M}$ mefenamic acid $(n=3)(\mathrm{A})$; for $\mathrm{K} 41 \mathrm{C}, \mathrm{V}_{1 / 2}=17.1 \mathrm{mV}$ and $k=19.7 \mathrm{mV}$ in control $(n=6), \mathrm{V}_{1 / 2}=11.3 \mathrm{mV}$ and $k=19.3 \mathrm{mV}$ in $100 \mu \mathrm{M}$ mefenamic acid $(n=4)$, and $\mathrm{V}_{1 / 2}=14.0 \mathrm{mV}$ and $k=18.3 \mathrm{mV}$ in $1 \mathrm{mM}$ mefenamic acid $(n=4)(\mathrm{B})$; and for $\mathrm{K} 41 \mathrm{R}, \mathrm{V}_{1 / 2}=72.7 \mathrm{mV}$ and $k=20.8 \mathrm{mV}$ in control $(n=3)$, and $\mathrm{V}_{1 / 2}=26.6 \mathrm{mV}$ and $k=49.3 \mathrm{mV}$ in $100 \mu \mathrm{M}$ mefenamic acid $(n=4)(\mathrm{C})$ (Table 2). (D) Change in $\mathrm{V}_{1 / 2}\left(\Delta \mathrm{V}_{1 / 2}\right)$ for each EQ $I_{K s}$ mutant in control versus mefenamic acid $(n=3-6$ at each concentration). $* P<0.03, * * P=0.0011$, $* * * P=0.0003$, and $* * * * P<0.0001$ (significant change in $\mathrm{V}_{1 / 2}$ comparing control to the presence of mefenamic acid). 
15 seconds (data not shown). Additionally, mefenamic acid also resulted in a significant leftward shift in the $V_{1 / 2}$ of activation of $\mathrm{K} 41 \mathrm{R}\left(\Delta \mathrm{V}_{1 / 2}:-46.2 \mathrm{mV}\right)$ (Fig. 7D; Table 2$)$. These results clearly show that residue $\mathrm{K} 41$, and especially the charge on this residue, is important in facilitating mefenamic acid's modulation of $I_{K s}$ gating kinetics.

\section{Discussion}

Previous studies have shown that mefenamic acid increases activation of $I_{K s}$ current and slows tail current decay, but curiously, all of these have only used a single concentration of drug $(100 \mu \mathrm{M})$. Generally, mefenamic acid effects are restricted to complexes of KCNQ1 and KCNE1 (Busch et al., 1994, 1997; Unsöld et al., 2000), although Abitbol et al. (1999) suggested that mefenamic acid may also facilitate KCNQ1 expressed alone in oocytes. Most studies have not shown that facilitation of $I_{K s}$ is accompanied by dramatic changes in the current activation time course, except for Unsöld et al. (2000). In the current study, we confirm that mefenamic acid enhances $I_{K s}$ activity (Fig. 1C) but not KCNQ1 alone (Fig. 5A), and that this effect is specific to $I_{K s}$ given that the large instantaneous current was blocked by HMR1556 (Fig. 1D), and no effect on endogenous currents was seen in untransfected cells treated with increasing concentrations of mefenamic acid (Fig. 1B). Following validation of these previous findings, we further defined the concentration dependence, effect of interpulse interval, stoichiometry dependence, effect on single-channel conductance, and the KCNE1 regulatory sites for mefenamic acid actions on $I_{K s}$.

Mefenamic Acid Actions on Saturated Complexes of $\boldsymbol{I}_{\boldsymbol{K} \boldsymbol{s}}(\mathbf{E Q})$. Increasing concentrations of mefenamic acid (1, 10, and $30 \mu \mathrm{M}$ ) have been used to confirm the functional effect of activating $I_{K s}$ in preconstricted rat mesenteric arteries $\left(\mathrm{E}_{\max }\right.$ of $96.1 \%$ ) (Chadha et al., 2012), but as noted earlier, regardless of the expression system, a single concentration of $100 \mu \mathrm{M}$ has been used to characterize the electrophysiological actions of mefenamic acid on $I_{K s}$. One striking effect is the induction of instantaneous current and reduction in the overall timedependent slow activation of $I_{K s}$ (Fig. 1C). These current changes were used to define the concentration dependence of mefenamic effects on $I_{K s}$, giving an $\mathrm{EC}_{50}$ and $n^{H}$ of $60 \mu \mathrm{M}$ and 0.49 , respectively (Fig. 2). An $n^{H}$ of $<1$ does not suggest multiple sites of action or positive cooperative binding of mefenamic acid to the channel complex, which is supported by the similar values of $n^{H}$ for different stoichiometric ratios of KCNQ1:KCNE1 ( $n^{H}=0.47$ and 0.66 for EQQ and EQQQQ, respectively). The more commonly reported (in all prior studies) action of mefenamic acid is to slow current deactivation (Busch et al., 1994; Magyar et al., 2006; Toyoda et al., 2006), and our experiments also confirmed the marked slowing of tail currents with time constants increasing across the range of repolarizing potentials (e.g., from 0.52 to 1.24 seconds at $-90 \mathrm{mV}$; Fig. $4 \mathrm{C}$ ).

Mefenamic acid has a hyperpolarizing effect on the $I_{K s} \mathrm{I}-\mathrm{V}$ and G-V relationships, which, in turn, is reflected by a leftward shift in the $\mathrm{V}_{1 / 2}$ of activation. Quantitatively, this hyperpolarization of the $\mathrm{V}_{1 / 2}$ has previously been inconsistently reported $(-15 \mathrm{mV}$ in $\mathrm{CHO}$ cells and $-26 \mathrm{mV}$ in canine ventricular myocytes) (Unsöld et al., 2000; Magyar et al., 2006). In Fig. 3, we demonstrated that this inconsistency may be related to mefenamic acid's striking effect on the G-V relationship that results in part from slowed channel deactivation at shorter pulse intervals. Prior studies often did not state intervals between pulses, so this cannot be verified.

With a voltage protocol that lasted 5 seconds and an interpulse interval of 7 seconds, there was not enough time to allow for complete $I_{K s}$ deactivation between voltage clamp pulses in the presence of mefenamic acid. As such, an accumulation of current occurred, which partly explains the dramatically altered G-V relationship and instantaneous current at this rate (Fig. $3 \mathrm{E}$ ). We initially hypothesized that if enough time were given to allow for $I_{K s}$ deactivation, the G-V relationship in the presence of mefenamic acid would mirror that seen in control (in the absence of mefenamic acid). However, even with an interpulse interval of 30 seconds in the presence of mefenamic acid, when deactivation should be complete, the G-V relationship still showed a significantly large departure in shape, slope, and $\mathrm{V}_{1 / 2}$ when compared with control. At interpulse intervals of 15,20 , and especially 30 seconds, there is a flattening of the voltage dependence of the G-V relationship caused by mefenamic acid that suggests a fundamental modification in the way that $I_{K s}$ senses and/or responds to changes in the transmembrane potential in the presence of the drug.

The single-channel data provide support for the whole-cell findings. There was no change in maximum channel conductance (Fig. 6), but openings did group toward higher open subconductance levels (Fig. 6, A and C) (Werry et al., 2013), which points to enhanced channel activation gating in the presence of mefenamic acid. In addition, data showed a large reduction in the first latency to opening of single channels, with channels opening in mefenamic acid de novo, soon after depolarizing pulses were applied. It is important to note that single-channel recordings did not indicate that failure of deactivation between pulses was an important factor in the decreased latency to opening (Fig. 6). Thus, single-channel kinetics provided significant insight into the changes induced by mefenamic acid seen at the whole-cell level. Finally, persistent single-channel openings were seen during repolarizations to $-40 \mathrm{mV}$ in the presence of mefenamic acid (Fig. 6A), mirroring the slow decay of macroscopic tail currents in the presence of drug (Fig. 4B).

To test whether actions of mefenamic acid on activation voltage gating could be sufficient to account for the current changes observed, we used an $I_{K s}$ model (Zaydman et al., 2014; Westhoff et al., 2019) to simulate drug action, as described in the Materials and Methods. Increasing VS forward rates for both activation transitions could account quantitatively for the dose- and rate-dependent action of mefenamic acid (Fig. 8). Increasing drug concentration caused the appearance of an instantaneous activating current with a subsequent slower phase, as seen in experimental data (Figs. 1 and 2). At the highest concentrations, the slow activating phase of current was almost abolished in simulations (Fig. 8A). The normalized-response relationship was simulated at a range of drug concentrations and was fit to a Hill equation with an $\mathrm{EC}_{50}$ of $78.6 \mu \mathrm{M}$, close to that found experimentally $(60 \mu \mathrm{M})$, and with a Hill slope of 0.81 , compared with $0.49-0.66$ found for EQ and EQQQQ, respectively. The action of mefenamic acid was also simulated for step voltage clamp pulses from -80 to $+100 \mathrm{mV}$ given every 7 and 15 seconds (Fig. 8, C and D). The complete set of simulated currents during the increasing amplitude clamp steps for all four conditions is shown 
in Fig. 8C, with the control tracings in blue and mefenamic acid in red. The 7 -second interval protocol lasts $\sim 130$ seconds in total, and the longer 15-second interval protocol lasts $\sim 285$ seconds. The effect of mefenamic acid was to increase peak current at both rates, especially at more negative potentials, as can be clearly seen. The tail currents from these protocols plotted as a function of voltage (Fig. 8D) give G-V relations, and as seen experimentally (Fig. $3 \mathrm{E}$ ), the effect of rate was to cause a minor slope change under control conditions, but a large hyperpolarizing shift was seen in the presence of mefenamic acid, which was greater at 7 seconds than at 15 seconds.

Mefenamic Acid Action Is Diminished with Fewer KCNE1 Subunits in the $I_{K s}$ Channel Complex. As the KCNQ1:KCNE1 stoichiometry of $I_{K s}$ is likely variable in vivo (Dvir et al., 2014), understanding the effect of mefenamic acid on different subunit ratios is extremely important, and the use of fixed stoichiometry constructs in the present experiments allowed a quantitative comparison of the action of mefenamic acid on different stoichiometries of $I_{K s}$ (Murray et al., 2016). The $\mathrm{V}_{1 / 2}$ of activation for KCNQ1 alone was not altered by mefenamic acid, but with the partially saturated $I_{K s}$ complexes, EQQ and EQQQQ, the $\mathrm{V}_{1 / 2}$ of activation was significantly hyperpolarized (Fig. 5). This hyperpolarization was less dramatic than that seen when $I_{K s}$ was fully saturated (EQ; Fig. 3E). Similarly, in the dose-response curves, the responses of EQQ and EQQQQ to mefenamic acid at all concentrations

A

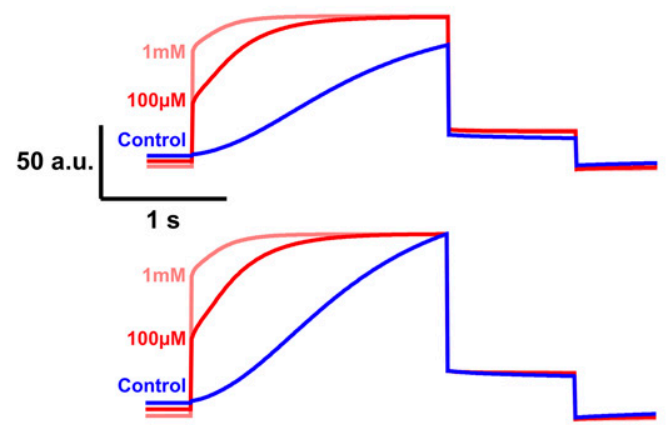

C

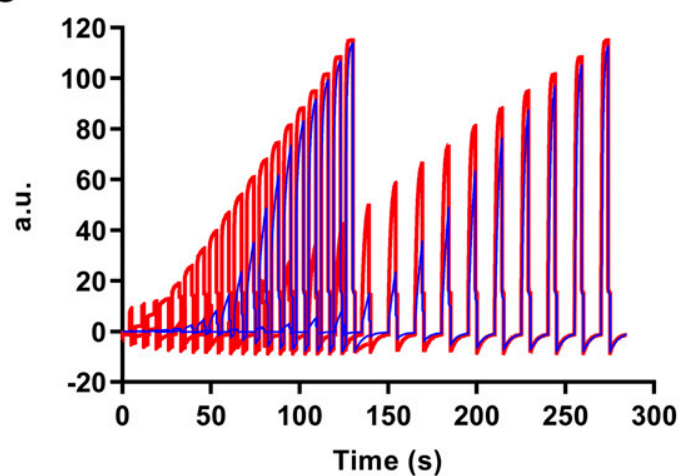

were significantly less than that of $\mathrm{EQ}$, which further supports the idea that the effect of mefenamic acid on $I_{K s}$ is stoichiometrically graded.

Mefenamic Acid Binding to the $\boldsymbol{I}_{K s}$ Complex. The binding site for DIDS and mefenamic acid on $I_{K s}$ has previously been suggested to lie between residues 39 and 43 on KCNE1, with residue E43 specifically identified as critical for the binding of DIDS (Abitbol et al., 1999). Whether this site and/or other residues in the mapped region are critical for the binding of mefenamic acid to KCNE1, and/or $I_{K s}$, was not studied. Through mutational analysis, we now show that, although mutation of residues E43 and L42 in KCNE1 results in a reduced response to mefenamic acid, residue $\mathrm{K} 41$ is critical for the action of mefenamic acid (Fig. 7).

Most of the mutations themselves do have variable effects on the gating of $I_{K s}$ in the absence of mefenamic acid (Table 2), but importantly, K41C has little effect on the position or slope of the $I_{K s}$ G-V relationship compared with WT EQ. This suggests that $\mathrm{K} 41 \mathrm{C}$ does not itself destabilize the normal interactions between KCNQ1 and KCNE1 in the $I_{K s}$ channel complex, or its ability to respond to applied changes in potential. These results are particularly interesting when taken in the context of the known importance of interactions between KCNQ1 and this region of KCNE to the pathophysiology of short QT syndrome, in which extremely slow deactivation is a feature (Dvir et al., 2014).

B
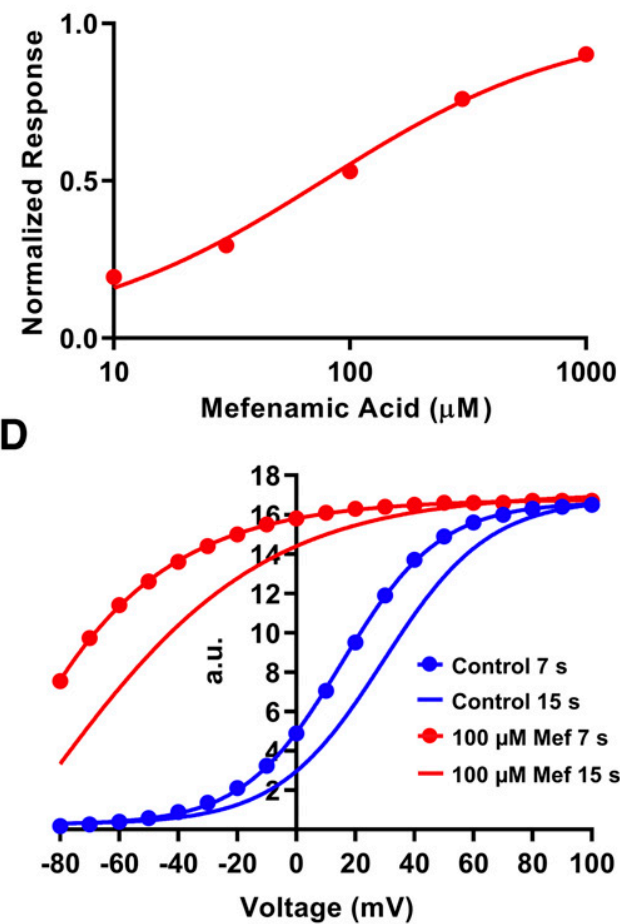

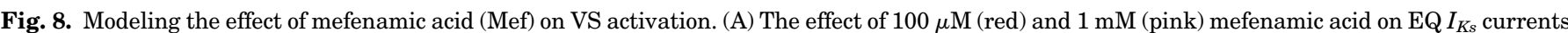

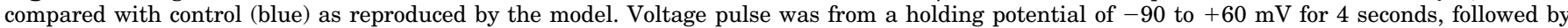

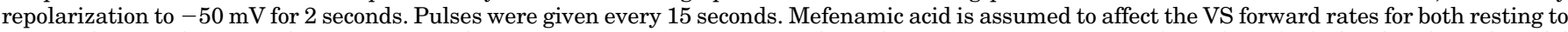

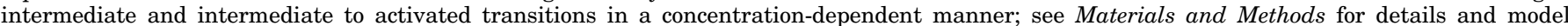

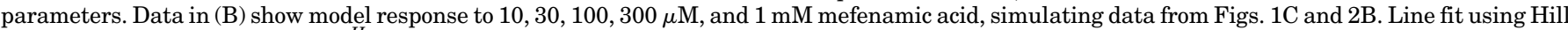

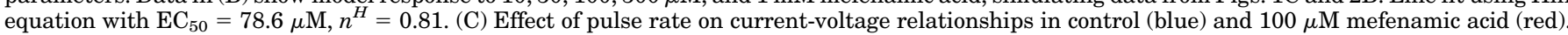

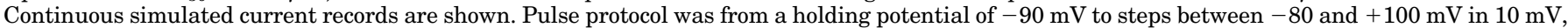

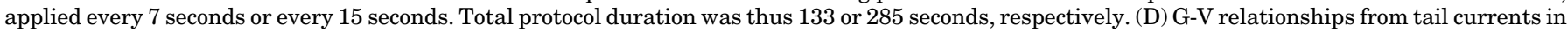

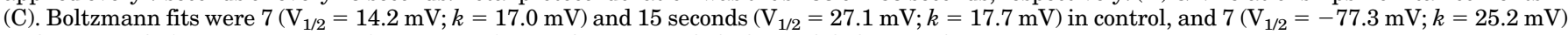
and 15 seconds $\left(\mathrm{V}_{1 / 2}=-50.8 \mathrm{mV} ; k=26.1 \mathrm{mV}\right)$ in mefenamic acid. Ordinate label a.u., arbitrary units. 
The E43C, L42C, and K41E mutations shift the $\mathrm{V}_{1 / 2}$ of $I_{K s}$ to approximately $+70 \mathrm{mV}$ (Table 2), which is opposite to the direction expected if they were inhibiting the interactions between KCNQ1 and KCNE1 (Murray et al., 2016), but which may explain their lesser response to mefenamic acid (Fig. 7D). The G40C mutant responds almost like WT to mefenamic acid (Supplemental Fig. 1), and so defines a proximal limit of the critical region. Taken together, the data indicate the primary importance of $\mathrm{K} 41$ in the binding of mefenamic acid to the $I_{K s}$ channel complex, and the response of $\mathrm{K} 41 \mathrm{R}$, but not $\mathrm{K} 41 \mathrm{E}$, to mefenamic acid suggests the importance of electrostatic rather than steric interactions in this effect.

Relevance of Mefenamic Acid Activation of $I_{K s}$ Channel Currents. Unlike most other activators that have little effect on $I_{K s}$ channels with increasingly saturated stoichiometries (Magyar et al., 2006; Gao et al., 2008; Yu et al., 2013), we have shown that mefenamic acid can enhance all $I_{K s}$ channel complexes of different stoichiometries, suggesting that molecules like it may represent a therapeutic approach to treating LQTS types 1 and 5. Although this is well beyond the scope of the present study, we note that mefenamic acid is presently prescribed at a recommended dosage of $500 \mathrm{mg} /$ day, which has been reported to equate to a mean plasma concentration of $82.9 \mu \mathrm{M}$ (Cryer and Feldman, 1998). This provides little clue toward the amount needed therapeutically to treat LQTS, but in our experiments such concentrations cause a potent activating effect of mefenamic acid that is also dependent on the stimulus rate, which is important because $I_{K s}$ primarily contributes to cardiac repolarization at high heart rates. The definitive stoichiometry of $I_{K s}$ in humans as well as the degree of $I_{K s}$ channel activation required for a therapeutically beneficial shortening of the QT interval are presently unknown, so we cannot know whether compounds such as mefenamic acid could have a beneficial effect in LQTS. We do, however, know that, due to the known adverse gastrointestinal effects of COX1 inhibition and block of other channels, such as TRPC, TRPM, and TREK channels (Takahira et al., 2005; Klose et al., 2011; Jiang et al., 2012), mefenamic acid itself is unlikely to be a suitable candidate.

\section{Conclusion}

The KCNQ1 channel alone is insensitive to up to $1 \mathrm{mM}$ mefenamic acid, and the drug increases $I_{K s}$ channel complex currents dependent upon the number of KCNE1 subunits present, unlike most other activators that have little effect on $I_{K s}$ channels with increasingly saturated stoichiometries. Single-channel studies reveal no change in the maximum conductance, so the instantaneous currents in the presence of mefenamic acid and the prolonged deactivation of tail currents are caused by a voltage-dependent shift of channel gating kinetics toward more negative potentials and a marked decrease in the voltage sensitivity of the channel. A shift to occupancy of higher subconductance states may also be partly responsible for the increase in peak currents. In silico modeling of the action of mefenamic acid showed that modulation of VS forward rates could account quantitatively for the drug effects. The ability of mefenamic acid to mediate these gating changes relies on binding to $I_{K s}$ regulated through K41 on KCNE1 and potentially other residues surrounding it. The presence of mefenamic acid bound to the $I_{K s}$ channel complex fundamentally alters the ability of the
KCNQ1/KCNE1 gating machinery to respond to the transmembrane potential gradient.

\section{Acknowledgments}

We thank Fariba Ataei and Emely Thompson for their technical assistance in cell culture (F.A.) and generation of KCNE1 mutants (E.T., F.A.).

\section{Authorship Contributions}

Participated in research design: Wang, Eldstrom, Fedida.

Conducted experiments: Wang, Eldstrom.

Performed data analysis: Wang, Eldstrom, Fedida.

Wrote or contributed to the writing of the manuscript: Wang, Eldstrom, Fedida.

\section{References}

Abbott GW (2014) Biology of the KCNQ1 potassium channel. New J Sci 2014:1-26. Abitbol I, Peretz A, Lerche C, Busch AE, and Attali B (1999) Stilbenes and fenamates rescue the loss of $\mathrm{I}(\mathrm{KS})$ channel function induced by an LQT5 mutation and other IsK mutants. EMBO $J$ 18:4137-4148.

Bendahhou S, Marionneau C, Haurogne K, Larroque MM, Derand R, Szuts V, Escande D, Demolombe S, and Barhanin J (2005) In vitro molecular interactions and distribution of KCNE family with KCNQ1 in the human heart. Cardiovasc Res 67:529-538.

Busch AE, Busch GL, Ford E, Suessbrich H, Lang HJ, Greger R, Kunzelmann K, Attali B, and Stühmer W (1997) The role of the IsK protein in the specific pharmacological properties of the IKs channel complex. Br J Pharmacol 122:187-189.

Busch AE, Herzer T, Wagner CA, Schmidt F, Raber G, Waldegger S, and Lang F (1994) Positive regulation by chloride channel blockers of IsK channels expressed in Xenopus oocytes. Mol Pharmacol 46:750-753.

Chadha PS, Zunke F, Davis AJ, Jepps TA, Linders JT, Schwake M, Towart R, and Greenwood IA (2012) Pharmacological dissection of $\mathrm{K}(\mathrm{v}) 7.1$ channels in systemic and pulmonary arteries. Br J Pharmacol 166:1377-1387.

Colquhoun D and Hawkes AG (1995) A Q-matrix cookbook how to write only one program to calculate the single-channel and macroscopic predictions for any kinetic mechanism, in Single-Channel Recording, 2nd ed. (Sakmann B and Neher E eds) pp 589-633, Plenum, New York.

Cryer B and Feldman M (1998) Cyclooxygenase-1 and cyclooxygenase-2 selectivity of widely used nonsteroidal anti-inflammatory drugs. Am J Med 104:413-421.

Doolan GK, Panchal RG, Fonnes EL, Clarke AL, Williams DA, and Petrou S (2002) Fatty acid augmentation of the cardiac slowly activating delayed rectifier current (IKs) is conferred by hminK. FASEB $J$ 16:1662-1664.

Dvir M, Peretz A, Haitin Y, and Attali B (2014) Recent molecular insights from mutated IKS channels in cardiac arrhythmia. Curr Opin Pharmacol 15:74-82.

Eldstrom $J$ and Fedida D (2011) The voltage-gated channel accessory protein KCNE2: multiple ion channel partners, multiple ways to long QT syndrome. Expert Rev Mol Med 13:e38.

Eldstrom J, Wang Z, Werry D, Wong N, and Fedida D (2015) Microscopic mechanisms for long QT syndrome type 1 revealed by single-channel analysis of $\mathrm{I}(\mathrm{Ks})$ with S3 domain mutations in KCNQ1. Heart Rhythm 12:386-394.

Gao Z, Xiong Q, Sun H, and Li M (2008) Desensitization of chemical activation by auxiliary subunits: convergence of molecular determinants critical for augmenting KCNQ1 potassium channels. J Biol Chem 283:22649-22658.

Gögelein H, Brüggemann A, Gerlach U, Brendel J, and Busch AE (2000) Inhibition of IKs channels by HMR 1556. Naunyn Schmiedebergs Arch Pharmacol 362: $480-488$

Hedley PL, Jørgensen P, Schlamowitz S, Wangari R, Moolman-Smook J, Brink PA, Kanters JK, Corfield VA, and Christiansen M (2009) The genetic basis of long QT and short QT syndromes: a mutation update. Hum Mutat 30:1486-1511.

Jiang H, Zeng B, Chen GL, Bot D, Eastmond S, Elsenussi SE, Atkin SL, Boa AN, and Xu SZ (2012) Effect of non-steroidal anti-inflammatory drugs and new fenamate analogues on TRPC4 and TRPC5 channels. Biochem Pharmacol 83: 923-931.

Klose C, Straub I, Riehle M, Ranta F, Krautwurst D, Ullrich S, Meyerhof W, and Harteneck C (2011) Fenamates as TRP channel blockers: mefenamic acid selectively blocks TRPM3. Br J Pharmacol 162:1757-1769.

Liin SI, Barro-Soria R, and Larsson HP (2015) The KCNQ1 channel - remarkable flexibility in gating allows for functional versatility. J Physiol 593:2605-2615.

Lundquist AL, Manderfield LJ, Vanoye CG, Rogers CS, Donahue BS, Chang PA, Drinkwater DC, Murray KT, and George AL Jr (2005) Expression of multiple KCNE genes in human heart may enable variable modulation of I(Ks). J Mol Cell Cardiol 38:277-287.

Magyar J, Horváth B, Bányász T, Szentandrássy N, Birinyi P, Varró A, Szakonyi Z Fülöp F, and Nánási PP (2006) L-364,373 fails to activate the slow delayed rectifier $\mathrm{K}+$ current in canine ventricular cardiomyocytes. Naunyn Schmiedebergs Arch Pharmacol 373:85-89.

Manderfield LJ and George AL Jr (2008) KCNE4 can co-associate with the I(Ks) (KCNQ1-KCNE1) channel complex. FEBS $J$ 275:1336-1349.

Morin TJ and Kobertz WR (2008) Counting membrane-embedded KCNE betasubunits in functioning $\mathrm{K}+$ channel complexes. Proc Natl Acad Sci USA 105: 1478-1482.

Mruk K and Kobertz WR (2009) Discovery of a novel activator of KCNQ1-KCNE1 K channel complexes. PLoS One 4:e4236. 
Murray CI, Westhoff M, Eldstrom J, Thompson E, Emes R, and Fedida D (2016) Unnatural amino acid photo-crosslinking of the IKs channel complex demonstrates a KCNE1:KCNQ1 stoichiometry of up to 4:4. eLife 5 :e11815.

Nakajo K, Ulbrich MH, Kubo Y, and Isacoff EY (2010) Stoichiometry of the KCNQ1 KCNE1 ion channel complex. Proc Natl Acad Sci USA 107:18862-18867.

Plant LD, Xiong D, Dai H, and Goldstein SA (2014) Individual IKs channels at the surface of mammalian cells contain two KCNE1 accessory subunits. Proc Nat Acad Sci USA 111:E1438-E1446.

Sanguinetti MC, Curran ME, Zou A, Shen J, Spector PS, Atkinson DL, and Keating MT (1996) Coassembly of K(V)LQT1 and minK (IsK) proteins to form cardiac I(Ks) potassium channel. Nature 384:80-83.

Santiago-Castillo JA, Covarrubias M, Sánchez-Rodríguez JE, Perez-Cornejo P, and Arreola J (2010) Simulating complex ion channel kinetics with IonChannelLab. Channels (Austin) 4:422-428.

Splawski I, Shen J, Timothy KW, Lehmann MH, Priori S, Robinson JL, Moss AJ, Schwartz PJ, Towbin JA, Vincent GM, et al. (2000) Spectrum of mutations in longQT syndrome genes. KVLQT1, HERG, SCN5A, KCNE1, and KCNE2. Circulation 102:1178-1185.

Takahira M, Sakurai M, Sakurada N, and Sugiyama K (2005) Fenamates and diltiazem modulate lipid-sensitive mechano-gated $2 \mathrm{P}$ domain $\mathrm{K}(+)$ channels. Pflugers Arch 451:474-478.

Thompson E, Eldstrom J, Westhoff M, McAfee D, Balse E, and Fedida D (2017) cAMPdependent regulation of $I_{K s}$ single-channel kinetics. J Gen Physiol 149:781-798.

Toyoda F, Ueyama H, Ding WG, and Matsuura H (2006) Modulation of functional properties of KCNQ1 channel by association of KCNE1 and KCNE2. Biochem Biophys Res Commun 344:814-820.

Unsöld B, Kerst G, Brousos H, Hübner M, Schreiber R, Nitschke R, Greger R, and Bleich M (2000) KCNE1 reverses the response of the human $\mathrm{K}+$ channel
KCNQ1 to cytosolic $\mathrm{pH}$ changes and alters its pharmacology and sensitivity to temperature. Pflugers Arch 441:368-378.

Werry D, Eldstrom J, Wang Z, and Fedida D (2013) Single-channel basis for the slow activation of the repolarizing cardiac potassium current, I(Ks). Proc Natl Acad Sci USA 110:E996-E1005.

Westhoff M, Eldstrom J, Murray CI, Thompson E, and Fedida D (2019) $I_{K_{s}}$ ionchannel pore conductance can result from individual voltage sensor movements. Proc Natl Acad Sci USA 116:7879-7888.

Westhoff M, Murray CI, Eldstrom J, and Fedida D (2017) Photo-cross-linking of $\mathrm{I}_{\mathrm{K}}$ demonstrates state-dependent interactions between KCNE1 and KCNQ1. Biophys $J$ 113:415-425.

Yu H, Lin Z, Mattmann ME, Zou B, Terrenoire C, Zhang H, Wu M, McManus OB Kass RS, Lindsley CW, et al. (2013) Dynamic subunit stoichiometry confers a progressive continuum of pharmacological sensitivity by KCNQ potassium channels Proc Natl Acad Sci USA 110:8732-8737.

Zaydman MA, Kasimova MA, McFarland K, Beller Z, Hou P, Kinser HE, Liang H, Zhang G, Shi J, Tarek M, et al. (2014) Domain-domain interactions determine the gating, permeation, pharmacology, and subunit modulation of the IKs ion channel. eLife 3:e3606.

Zheng Y, Zhu X, Zhou P, Lan X, Xu H, Li M, and Gao Z (2012) Hexachlorophene is a potent KCNQ1/KCNE1 potassium channel activator which rescues LQTs mutants. PLoS One 7:e51820.

Address correspondence to: Dr. David Fedida, Life Sciences Centre, 2350 Health Sciences Mall, Room 2.301, Vancouver, British Columbia V6T 1Z3, Canada. E-mail: david.fedida@ubc.ca 